\title{
Analysis of Correlations and their Impact on Convergence Rates in Monte Carlo Eigenvalue Simulations
}

\author{
J. Miao ${ }^{\mathrm{a}}$, B. Forget ${ }^{\mathrm{a}}$, K. Smith ${ }^{\mathrm{a}}$ \\ ${ }^{a}$ Massachusetts Institute of Technology, 77 Massachusetts Ave, Cambridge, MA 02139
}

\begin{abstract}
This paper provides an analysis of the generation-to-generation correlations as observed when solving full core eigenvalue problems on PWR systems. Many studies have in the past looked at the impact of these correlations on reported variance and this paper extends the analysis to the observed convergence rate on the tallies, the effect of tally size and the effect of generation size. Since performing meaningful analysis on such a large problem is inherently difficult, a simple homogeneous reflective cube problem with analytical solution was developed that exhibits similar behavior to the full core PWR benchmark. The data in this problem was selected to match the dimensionality of the reactor problem and preserve the migration length traveled by neutrons. Results demonstrate that the variance will deviate significantly from the $1 / \mathrm{N}$ ( $\mathrm{N}$ being the number of simulated particles) convergence rate associated with truly independent generations, but will eventually asymptote to $1 / \mathrm{N}$ after 1000 's of generations regardless of the numbers of neutrons per generation. This indicates that optimal run strategies should emphasize lower number of active generations with greater number of neutrons per generation to produce the most accurate tally results. This paper also describes and compares three techniques to evaluate suitable confidence intervals in the presence of correlations, one based on using history statistics, one using generation statistics and one batching generations to reduce batch-to-batch correlation.
\end{abstract}

Keywords: Monte Carlo, Tally Convergence, Autocorrelation, Confidence Intervals

\section{Introduction}

Monte Carlo methods have long been considered a reference for neutron transport simulations since they make very limited approximations in simulating the random walk of neutrons in a system. Most often it is assumed that each neutron is independent of all others thus allowing for simple evaluation of unbiased means and uncorrelated variance. Previous work has observed correlation effects between neutrons in systems with fission, particularly when performing eigenvalue simulations based on the power iteration. Brissenden and Garlick [1] demonstrated the existence of this bias in the Monte Carlo power iteration and suggested that this bias became quite small when a sufficient number of neutrons per generation were simulated. Dumonteil et al 2] further studied this issue and attributed the generationto-generation correlation to spatial correlation of the fission process and ensuing asymmetry between neutron creation and annihilation in a stochastic branching process.

These works thus indicate that using a sufficiently large number of particles per generation will incur a negligible bias, but neglecting the correlations in the variance estimate will lead to an underestimation of that variance. Additionally, the existence of correlation impacts the convergence rate of the sample mean leading to the conundrum that additional generations of neutrons will only slightly improve the sample mean and may not be worth the additional run time. This has been demonstrated extensively in recent work by Herman on a realistic 2D full core PWR benchmark [3]. The simple approach to avoid underestimation of the variance is to perform multiple independent simulations with different initial random seeds. This will lead to good variance estimates but requires lots of additional work since each independent simulation needs an independent fission source and will not improve convergence rates [4. Many studies over the years have been performed to evaluate the ratio between estimated and

Email addresses: jlmiao@mit.edu (J. Miao), bforget@mit.edu (B. Forget), kord@mit.edu (K. Smith) 


\begin{tabular}{ccccccccc}
\hline \multicolumn{2}{c}{ Geometry } & \multirow{2}{*}{$\nu$} & \multicolumn{4}{c}{ Macro Cross-Section } & \multirow{2}{*}{$k_{\text {eff }}$} & Meshes \\
\cline { 1 - 5 } Boundary & Width $(\mathrm{cm})$ & & $\Sigma_{s}\left(\mathrm{~cm}^{-1}\right)$ & $\Sigma_{c}\left(\mathrm{~cm}^{-1}\right)$ & $\Sigma_{f}\left(\mathrm{~cm}^{-1}\right)$ & $\Sigma_{t}\left(\mathrm{~cm}^{-1}\right)$ & \\
\hline Reflective & 400 & 2.45 & 0.270 & 0.018 & 0.012 & 0.300 & 1 & $16 \times 16 \times 16$ \\
\hline
\end{tabular}

true variance. This eventually led to the concept of generations-per-batch [5] that can provide a better estimate of the variance in a single simulation.

In recent years, more effort has been dedicated at evaluating the magnitude and lag of the the autocorrelation coefficients observed in the eigenvalue mode of Monte Carlo simulations. Herman et al [4] calculated the autocorrelation coefficients and showed the dependency on the mesh size as well as the insensitivity to the number of neutrons per generation. Yamamoto, et al. 6] [7] used the autogressive (AR) model to predict underestimation of variance. In this method, the fission source distribution for each generation is expanded with eigenfunctions of the diffusion equation. Generation-to-generation correlation is represented by propagation of fluctuation of expansion coefficients. Sutton [8] explained and predicted underestimation of variance with a discretized phase space approach inspired by Brissenden and Garlick's work [1.

In this paper, we will demonstrate the impact of the autocorrelation coefficients on the sample mean and its convergence rate during the power iteration process and seek to develop some intuition on how best to assign computational resources in evaluating problems with large correlations starting from a stationary fission source. This work will use a very simple problem with known solution to eliminate any uncertainties that can occur in evaluating convergence rates. Related work was performed by Tuttelberg et al 9 ] were they optimized the number of neutrons per generation for a fixed amount of total neutrons on both inactive and active generations to minimize bias and statistical error. However, their work did not account explicitly for the impact of correlation effects on statistical error and convergence rate. In this work, we will focus on determining optimal simulation parameters for the active generations only in the presence of strong correlations as observed in full core simulations. It will thus be assumed that acceleration methods can be used in the inactive region [4] 10, 11] [12] [13] 14] [15] to provide an initial unbiased stationary source. Section 2 will present the simple mono-energetic homogeneous cube problem with reflective boundary conditions used in the simulation and demonstrate its similarities with previous analysis on a full core PWR. Section 3 will present a review on statistical analysis in the presence of correlation. Section 4 will develop the theory behind the autocorrelation coefficients and the variance estimates. Section 5 will derive the optimal relation between generations and number of neutrons per generation, followed in section 6 on the best practices to define interval estimates of tallied quantities. Concluding remarks and future work recommendations will follow in Section 7.

\section{Homogeneous Cube}

Analyzing correlation coefficients on full core realistic problems becomes a very costly endeavor. Herman et al 4 ] were able to compute such coefficients on the 2D BEAVRS benchmark using extensive computational time making substantial analysis very impractical. In order to accelerate the process a simple benchmark was developed that preserves the correlation effects, reduces run time and has a simple analytical reference solution. Parameters of the homogenized cubic reactor are given in Table 1. The simple benchmark was chosen as a $400 \mathrm{~cm}$ reflective cube since it has dimensionality similar to a full core PWR, additionally the cross sections were selected such that the system is critical and preserves the migration length of neutrons.

Previous work relied on obtaining a suitable reference solution which was quite costly and introduced some amount of uncertainty in the analysis. The simple benchmark used in this work alleviates this issue since the reference solution is known analytically, thus eliminating any possible aberration that can be observed when computing RMS convergence rates.

The Relative Square Error (RSE) between accumulated tallies and the reference source distribution 
is defined for each tally region $m$ as

$$
R S E_{m}=\frac{\left(\hat{X}_{m}-\left\langle X_{m}\right\rangle\right)^{2}}{\left\langle X_{m}\right\rangle^{2}}
$$

where $\hat{X}_{m}$ is the generation averaged estimator (i.e. within a single generation) and $\left\langle X_{m}\right\rangle$ is the reference solution. $R S E$ in $\mathrm{Eq} 2.1$ is then averaged over all $M$ tally regions and taken square root. We define this as Root Mean Square error (RMS) in Eq 2.2 $R M S$ is essentially a spatial average using $L_{2}$ norm.

$$
R M S=\sqrt{\frac{\sum_{m=1}^{M} R S E_{m}}{M}}
$$

The fission source distribution in the simple benchmark can be evaluated analytically, as shown by $\mathrm{Eq} 2.3$, and used as a reference in the $R M S$ calculation.

$$
\left\langle X_{m}\right\rangle=\frac{\Sigma_{f}}{\Sigma_{t}-\Sigma_{s}} \frac{1}{(\Delta x)^{3}}
$$

where $\Sigma_{t}, \Sigma_{f}, \Sigma_{s}$ are the total cross section, fission cross section, scattering cross section of the cube respectively and $(\Delta x)^{3}$ is the volume of each tally region.

To analyze this benchmark a simple Monte Carlo code was developed on a GPU to accelerate the analysis. At each generation the number of neutrons is normalized to the number of threads to be launched. Each thread has a local collision tally in each spatial bin and a reduction algorithm is performed after each generation to obtain the global tally. When the generation size (number of neutrons per generation) exceeds the number of threads on the GPU, kernels are launched sequentially. Generation sizes are selected to the power of 2 for more efficient use of the GPU hardware. The fission source distribution is then obtained by correcting the collision source distribution by the constant factor $\frac{\Sigma_{f}}{\Sigma_{t}}$.

A mesh tally of $16 \times 16 \times 16$ was selected since it is representative of an assembly size tally in a PWR. Fig 1(a) and Fig 2(a) illustrate the similarities in autocorrelation coefficients (ACC) between the 2D BEAVRS with assembly size tallies and the simple cube with $25 \mathrm{~cm}$ size tallies. The simple benchmark also illustrates an important feature of the problem, that of lower autocorrelation coefficients with smaller tally regions, as seen in the BEAVRS benchmark from figures 1(a) and 1(b) and illustrated in the benchmark by figures 2(a) and 2(b). Additionally, figures 1(c) and 2(c) present similar deviations from the ideal convergence rate which is directly caused by the presence of correlations.

\section{Background}

\subsection{Variance of correlated sample average}

In Monte Carlo eigenvalue simulations, quantities of interests are usually estimated as an average over many generations once a stationary fission source is obtained. The sample mean is reported accompanied by an estimate of the variance indicating the level of statistical uncertainty of the simulation and allowing for the definition of confidence intervals.

By definition, the variance of the sample mean is given by

$$
\operatorname{Var}[\bar{X}]=\operatorname{Var}\left[\frac{\sum_{i=1}^{N} X_{i}}{N}\right]=\frac{1}{N^{2}} \operatorname{Var}\left[\sum_{i=1}^{N} X_{i}\right]=\frac{1}{N^{2}} E\left[\left(\sum_{i=1}^{N} X_{i}-E\left[\sum_{i=1}^{N} X_{i}\right]\right)^{2}\right]
$$

where $X_{i}$ is the result obtained from generation $i$ and $N$ is the total number of active generations.

The commutability of expectation and sum decomposes the variance into two terms

$$
\begin{aligned}
\operatorname{Var}[\bar{X}] & =\frac{1}{N^{2}}\left(\sum_{i} E\left[\left(X_{i}-E\left[X_{i}\right]\right)^{2}\right]+\sum_{i \neq j} E\left[\left(X_{i}-E\left[X_{i}\right]\right)\left(X_{j}-E\left[X_{j}\right]\right)\right]\right) \\
& =\frac{1}{N^{2}}\left(N \sigma^{2}+2 \sum_{i<j} \operatorname{Cov}\left[X_{i}, X_{j}\right]\right)
\end{aligned}
$$




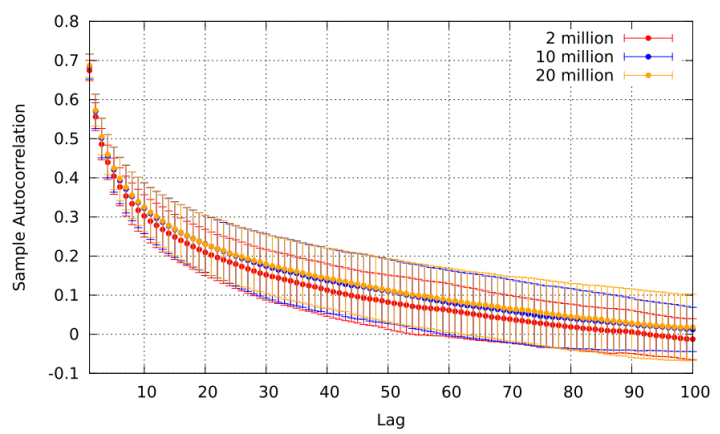

(a) Autocorrelation coefficients assembly-size tally

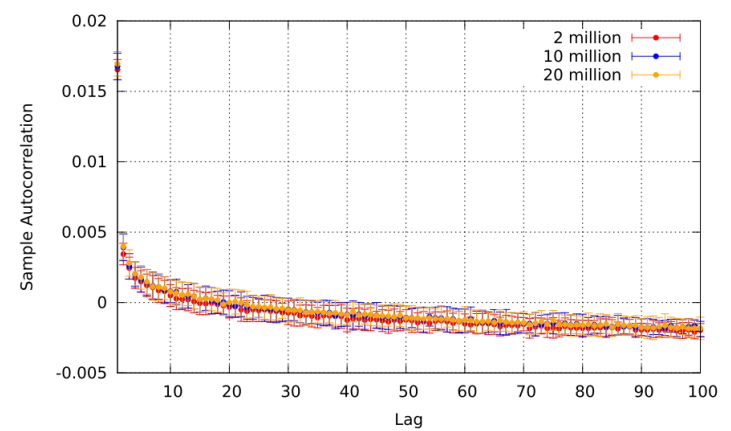

(b) Autocorrelation coefficients pin-size tally

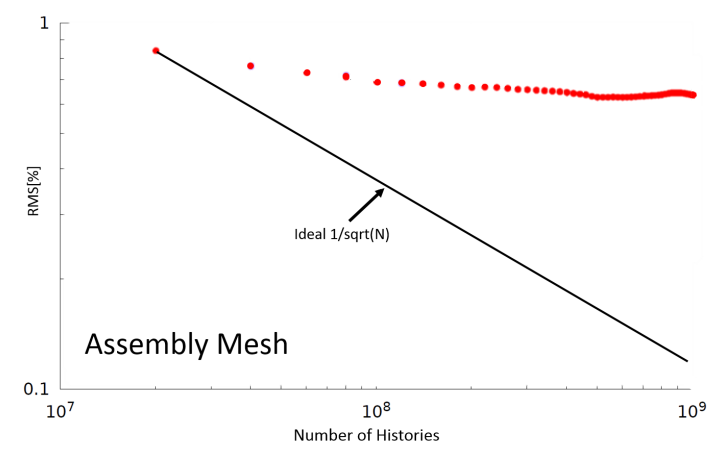

(c) RMS Convergence assembly-size tally

Figure 1: 2D BEAVRS autocorrelation coefficients and assembly-size tally convergence rate [4]

where $\sigma^{2}$ is the variance of $X$.

If the generations were uncorrelated, as often assumed in Monte Carlo simulations, the covariance term would vanish and the variance of the sample mean would be

$$
\operatorname{Var}[\bar{X}]=\frac{\sigma^{2}}{N}
$$

In the presence of correlation, since the generation tally values can be modeled as stationary time series, the covariance between two batches only depends on batch distance or lag, $k$, [16] [4]

$$
\begin{aligned}
\operatorname{Var}[\bar{X}] & =\frac{1}{N^{2}}\left(N \sigma^{2}+2 \sum_{k=1}^{N-1} \sum_{i=1}^{N-k} \operatorname{Cov}\left[X_{i}, X_{i+k}\right]\right) \\
& =\frac{1}{N^{2}}\left(N \sigma^{2}+2 \sum_{k=1}^{N-1}(N-k) \operatorname{Cov}\left[X_{i}, X_{i+k}\right]\right)
\end{aligned}
$$

Taking into account the covariance between generations, the variance of the sample mean of observable $X$ can be evaluated with $\mathrm{Eq} 3.5$. where $\sigma^{2}$ is the variance of $X, N$ is the number of active generations, and $\rho_{k}$ is the autocorrelation coefficient between $X$ 's with generation lag $k$.

$$
\begin{aligned}
\operatorname{Var}[\bar{X}] & =\frac{\sigma^{2}}{N}\left(1+2 \sum_{k=1}^{N-1}\left(1-\frac{k}{N}\right) \rho_{k}\right) \\
\rho_{k} & =\frac{\operatorname{Cov}\left[X_{i} X_{i+k}\right]}{\sqrt{\operatorname{Var}\left[X_{i}\right] \operatorname{Var}\left[X_{i+k}\right]}}
\end{aligned}
$$

As can be seen from $\mathrm{Eq} 3.5$, if the variance of the sample formed by the generation results $\left\{X_{1}, \cdots, X_{N}\right\}$ is divided by the total number of active generations $N$ to estimate the variance of their average, the 


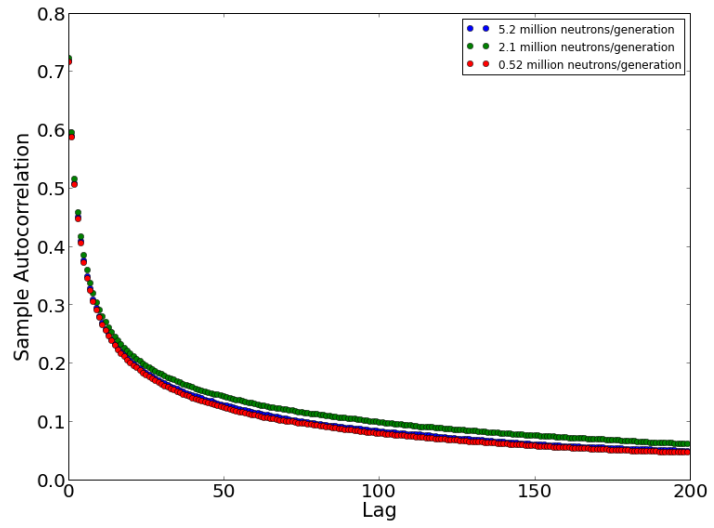

(a) Autocorrelation coefficients $16 \times 16 \times 16$ tally

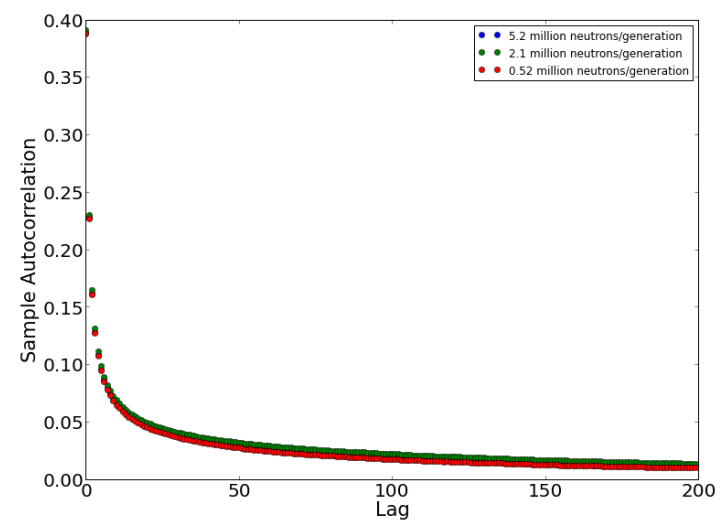

(b) Autocorrelation coefficients $32 \times 32 \times 32$ tally

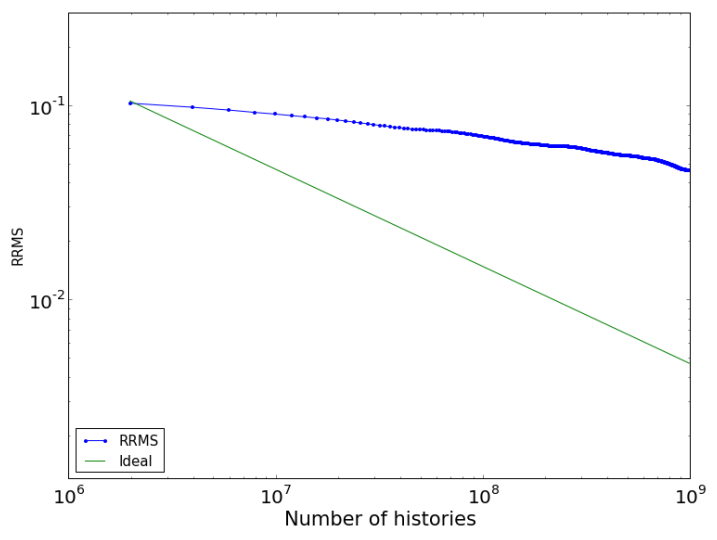

(c) RMS Convergence $16 \times 16 \times 16$ tally

Figure 2: Simple benchmark autocorrelation coefficients and assembly-size tally convergence rate 
variance of the estimator is underestimated by a factor $r(N)$ defined as

$$
r(N) \equiv 1+2 \sum_{k=1}^{N-1}\left(1-\frac{k}{N}\right) \rho_{k}
$$

When estimating observable $X$ (with expectation $\langle X\rangle$ ) by estimator $\hat{X}$, it can be shown that the expected square error (ESE) equals the variance of the variable plus the bias of the estimator.

$$
E S E=E\left[(\hat{X}-\langle X\rangle)^{2}\right]=\operatorname{Var}[\hat{X}]+(E[\hat{X}]-\langle X\rangle)^{2}
$$

If the estimator is unbiased, the expected square error becomes

$$
E S E=\operatorname{Var}[\bar{X}]
$$

Eq 3.9 shows the equivalence between square error and variance of estimator. Therefore, the error and convergence rate of the estimator (the sample mean) can be predicted by its ESE or variance. The $E S E$ can be used as a predictor for the $R M S$ as follows

$$
\begin{aligned}
E S E_{m} & =\operatorname{Var}\left[\bar{X}_{m}\right] \\
E\left[R S E_{m}\right] & =\frac{\operatorname{Var}\left[\bar{X}_{m}\right]}{\left\langle X_{m}\right\rangle^{2}} \\
R M S_{\text {predict }} & =\sqrt{\frac{\sum_{m=1}^{M} \frac{\operatorname{Var}\left[\bar{X}_{m}\right]}{\left\langle X_{m}\right\rangle^{2}}}{M}}=\sqrt{E\left[R M S^{2}\right]}
\end{aligned}
$$

The predicted $R M S$ can be expanded using the definition of variance of $\mathrm{Eq} 3.5$

$$
R M S_{\text {predict }}=\sqrt{\frac{1}{M} \sum_{m=1}^{M} \frac{\operatorname{Var}\left[\bar{X}_{m}\right]}{\left\langle X_{m}\right\rangle^{2}}}=\sqrt{\frac{1}{M N} \sum_{m=1}^{M} \frac{\sigma_{m}^{2}}{\left\langle X_{m}\right\rangle^{2}}\left(1+2 \sum_{k=1}^{N-1}\left(1-\frac{k}{N}\right) \rho_{m, k}\right)}
$$

By defining an average variance for all tally regions as

$$
\overline{\sigma^{2}}=\frac{1}{M} \sum_{m=1}^{M} \frac{\sigma_{m}^{2}}{\left\langle X_{m}\right\rangle^{2}}
$$

and an average variance-weighted ACC,

$$
\overline{\rho_{k}}=\frac{\sum_{m=1}^{M} \frac{\sigma_{m}^{2}}{\left\langle X_{m}\right\rangle^{2}} \rho_{m, k}}{\sum_{m=1}^{M} \frac{\sigma_{m}^{2}}{\left\langle X_{m}\right\rangle^{2}}}
$$

the predicted $R M S_{\text {predict }}$ can be cast in the same form as $\mathrm{Eq} 3.5$

$$
R M S_{\text {predict }}=\sqrt{\frac{\overline{\sigma^{2}}}{N}\left(1+2 \sum_{k=1}^{N-1}\left(1-\frac{k}{N}\right) \bar{\rho}_{k}\right)}
$$

With knowledge of the ACCs or a suitable approximation, this formula permits predicting the convergence rate of the tallies as a function of the number of generations, $N$, and the number of neutrons per generation, via the $\bar{\sigma}^{2}$ term. 


\subsection{Bias of Sample Variance Estimated from Generation Tallies}

Eq 3.16 shows that the variance, $\overline{\sigma^{2}}$, divided by the sample size, $N$, underestimates the variance of the mean. On the other hand, if the variance is unknown, it must be estimated from samples that are correlated. This section provides the correction to the variance estimator needed to take into account sample correlation. For a uncorrelated sample $\left\{X_{1}, \cdots, X_{n}\right\}$ the unbiased estimate of variance is

$$
\widehat{\sigma_{0}^{2}}=\frac{1}{N-1} \sum_{i=1}^{N}\left(X_{i}-\bar{X}\right)^{2}
$$

The bias of the above estimator for a correlated sample can be detected from its expectation,

$$
E\left[\widehat{\sigma_{0}^{2}}\right](N-1)=E\left[\sum_{i=1}^{N}\left(X_{i}-\bar{X}\right)^{2}\right]=N\left(E\left[X_{i}^{2}\right]-E\left[\bar{X}^{2}\right]\right)
$$

From definition of variance,

$$
E\left[\bar{X}^{2}\right]=E[\bar{X}]^{2}+\operatorname{Var}[\bar{X}]
$$

Expressing the right hand side in terms of $X_{i}$, knowing $E[\bar{X}]=E\left[X_{i}\right]$ and $\operatorname{Var}[\bar{X}]$ given in Eq 3.5, yields

$$
\begin{aligned}
E\left[\widehat{\sigma_{0}^{2}}\right](N-1) & =N\left(E\left[X_{i}^{2}\right]-E\left[X_{i}\right]^{2}-\frac{\operatorname{Var}\left[X_{i}\right]}{N}\left(1+2 \sum_{k=1}^{N-1}\left(1-\frac{k}{N}\right) \rho_{k}\right)\right) \\
& =N\left(\sigma^{2}-\frac{\sigma^{2}}{N}\left(1+2 \sum_{k=1}^{N-1}\left(1-\frac{k}{N}\right) \rho_{k}\right)\right)
\end{aligned}
$$

After re-organization,

$$
\frac{E\left[\widehat{\sigma_{0}^{2}}\right](N-1)}{N\left(1-\frac{1}{N}\left(1+2 \sum_{k=1}^{N-1}\left(1-\frac{k}{N}\right) \rho_{k}\right)\right)}=\sigma^{2}
$$

If $\rho_{k}$ is known, the denominator of $\mathrm{Eq} 3.21$ can be incorporated into the expectation operator. And after substituting the definition of $\widehat{\sigma_{0}^{2}}, \mathrm{Eq} 3.21$ yields

$$
E\left[\frac{\sum_{i=1}^{N}\left(X_{i}-\bar{X}\right)^{2}}{N-1-2 \sum_{k=1}^{N-1}\left(1-\frac{k}{N}\right) \rho_{k}}\right]=\sigma^{2}
$$

Inside the expectation operator of $\mathrm{Eq} 3.22$ is an unbiased estimator of the variance $\sigma^{2}$.

$$
\widehat{\sigma^{2}}=\frac{\sum_{i=1}^{N}\left(X_{i}-\bar{X}\right)^{2}}{N-1-2 \sum_{k=1}^{N-1}\left(1-\frac{k}{N}\right) \rho_{k}}
$$

Comparing $\widehat{\sigma^{2}}(\mathrm{Eq} 3.23)$ and $\widehat{\sigma_{0}^{2}}(\mathrm{Eq} 3.17)$ shows that the bias of the estimator $\widehat{\sigma_{0}^{2}}$ is related with the variance underestimation ratio $r$

$$
\widehat{\widehat{\sigma_{0}^{2}}}=\frac{N-r}{N-1}
$$

Fig 3 plots the ratio of sample variance calculated from Eq 3.17 to a reference with known ACCs for the simple cube benchmark on a $16 \times 16 \times 16$ mesh. It can be seen that the uncorrected sample variance $(\mathrm{Eq} 3.17)$ underestimates the real sample variance by around $10 \%$ even after 1000 generations. Variance estimated with $\mathrm{Eq} 3.17$ from a simulation containing 20,000 generations was taken as the reference by assuming $N=20,000 \gg r$. 


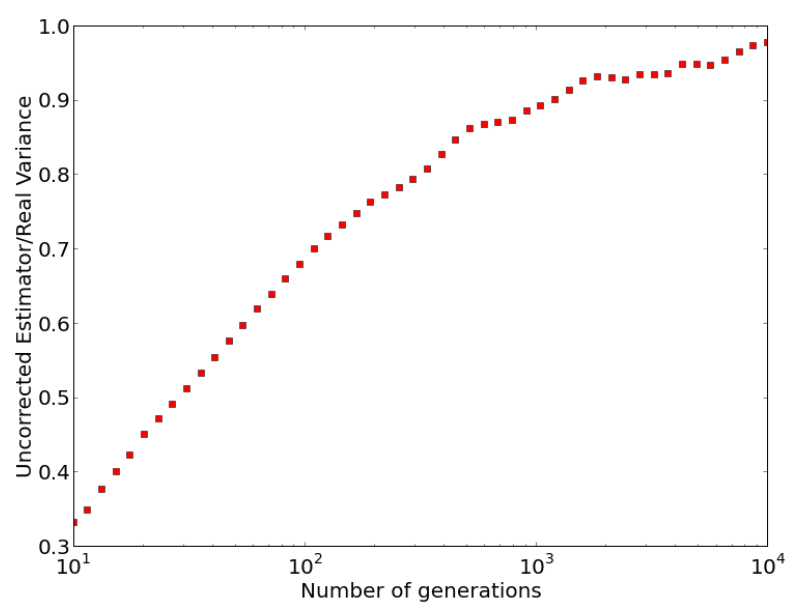

Figure 3: Bias of variance estimator

A similar problem exists for the estimate of autocorrelation coefficients $\rho_{k}(\mathrm{Eq}$ 3.6). The estimate is given in $\mathrm{Eq} 3.25$. 4 .

$$
\hat{\rho}_{k}=\frac{(N-k) \sum_{i=1}^{N-k} X_{i} X_{i+k}-\sum_{i=1}^{N-k} X_{i} \sum_{i=1}^{N-k} X_{i+k}}{\sqrt{(N-k) \sum_{i=1}^{N-k} X_{i}^{2}-\left(\sum_{i=1}^{N-k} X_{i}\right)^{2}} \sqrt{(N-k) \sum_{i=1}^{N-k} X_{i+k}^{2}-\left(\sum_{i=1}^{N-k} X_{i+k}\right)^{2}}}
$$

In order to calculate $\hat{\rho}_{k}, X_{i}$ and $X_{i+k}(i=1, \cdots, N-k)$ are treated as two separate data sets and $\rho_{k}$ is calculated as the normalized covariance between the two sets. Since two variance estimators appear in the denominator of $\hat{\rho}_{k}$ and we do not correct for them using the ACCs, in this analysis Eq 3.25 is used to evaluate $\rho_{k}$ for $k \ll N$.

\section{Correlation analysis}

\subsection{Fitting autocorrelation coefficients}

From the autocorrelation coefficients observed in section 2, it is reasonable to assume that the spatially averaged autocorrelation coefficients decay exponentially as a function of the generation lag [7]. This hypothesis can be further validated by observing that the tallies in a region are both Gaussian and Markovian (depends only on previous generation) and that such Gauss-Markov processes present exponential autocorrelation coefficients [17. Fig 4(a) shows the agreement between the fitted autocorrelation coefficient and sampled autocorrelation coefficient on the simple cube problem. The exponential decay assumption of spatially averaged ACC is expressed as

$$
\overline{\rho_{k}}=\sum_{j=1}^{J} c_{j} q_{j}^{k}
$$

where $J$ is the number of decay modes used to fit the ACC and $0<q_{j}<1$ since ACC decays as lag increases. Without loss of generality, the following analysis is based on the simple fit with $J=1$, but all conclusions still hold for any $J>1$. Using the simple exponential fit,

$$
\overline{\rho_{k}}=\rho_{0} q^{k}
$$

the sum $\mathrm{Eq} 3.7$ can be evaluated

$$
r=1+2 \rho_{0}\left(\frac{q}{1-q}-\frac{q^{2}-q^{N}}{(1-q)^{2} N}-\frac{q+q^{N}}{(1-q) N}\right)
$$




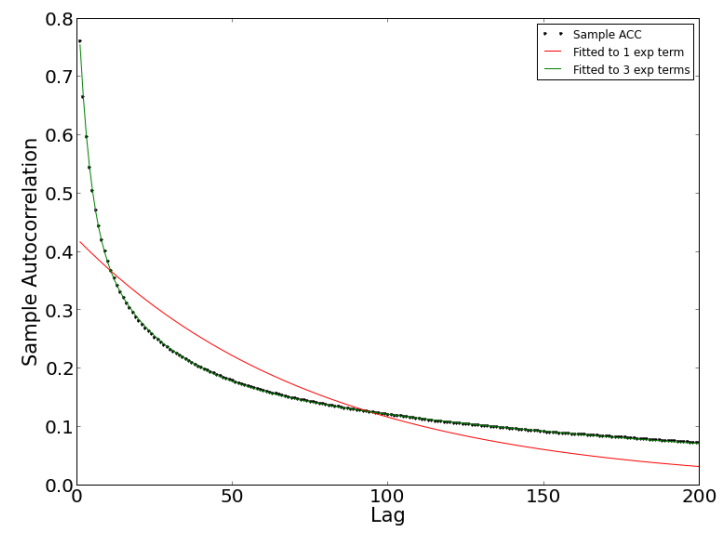

(a) Sample ACC and fitted ACC in the cube

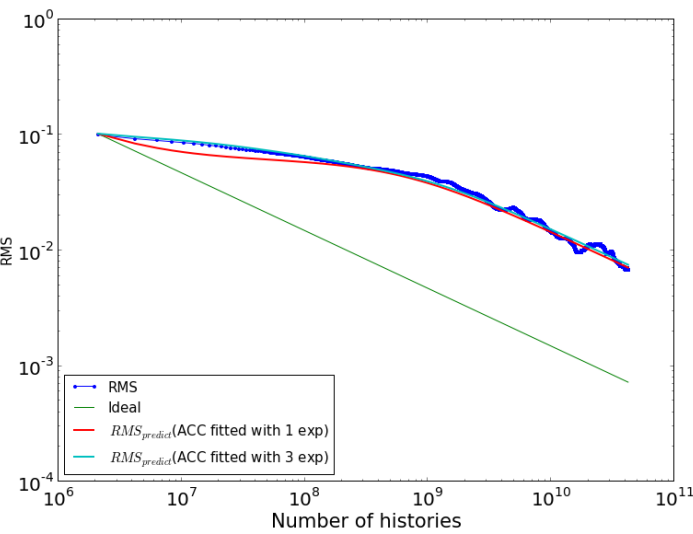

(b) $R M S$ and $R M S_{\text {predict }}$ from fitted ACC

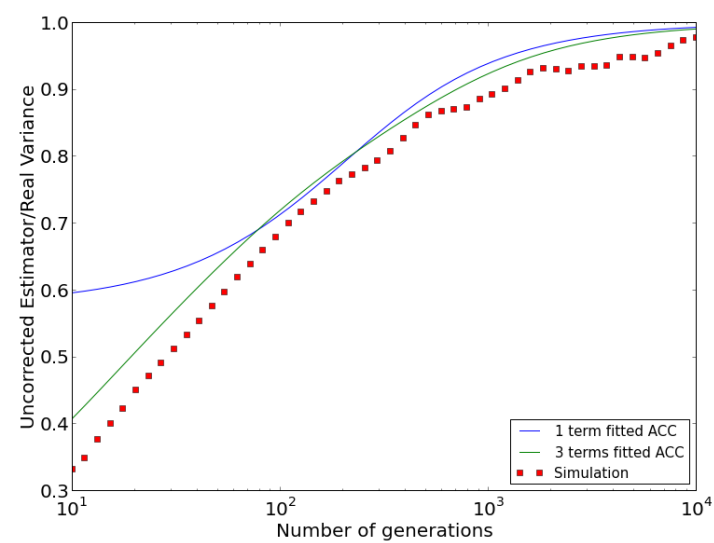

(c) Sample variance underestimate ratio and its prediction from fitted ACC

Figure 4: Verification of exponential ACC fitting 
$R M S_{\text {predict }}^{2}(\mathrm{Eq} 3.16)$ can be evaluated as a function of the number of generations $N$, spatially averaged variance $\sigma^{2}(\mathrm{Eq} 3.14)$, and the two ACC fitting parameters $\rho_{0}, q$,

$$
R M S_{\text {predict }}^{2}\left(N, \sigma^{2}, \rho_{0}, q\right)=\frac{\sigma^{2}}{N}\left[1+2 \rho_{0}\left(\frac{q}{1-q}-\frac{q^{2}-q^{N}}{(1-q)^{2} N}-\frac{q+q^{N}}{(1-q) N}\right)\right]
$$

As $N$ approaches infinity, only the first two terms in $R M S_{\text {predict }}^{2}$ remain, thus indicating the asymptotic $1 / N$ trend of $R M S_{\text {predict. }}^{2}$ It also shows that the asymptotic ratio of variance underestimation with uncorrelated variance $(\mathrm{Eq} 3.3)$ is equal to $1+2 \rho_{0} \frac{q}{1-q}$. The convergence rate of $R M S$ can be obtained from the asymptotic slope of $R M S_{\text {predict }}^{2}$ versus $N$ in the log-log scale.

$$
\lim _{N \rightarrow \infty} \frac{\partial \log \left(R M S_{\text {predict }}^{2}\right)}{\partial \log (N)}=\frac{N\left((q-1)^{2}-2 q \rho_{0}(q-1)\right)+O\left(q^{N}\right)+O\left(N q^{N}\right)+O(1)}{N\left(2 q \rho_{0}(q-1)-(q-1)^{2}\right)+O\left(q^{N}\right)+O(1)} \rightarrow-1
$$

Therefore, the asymptotic convergence rate of $R M S_{\text {predict }}^{2}$ follows $1 / N$, thus $R M S$ follows $1 / \sqrt{N}$ even in the presence of generation-to-generation correlation.

In addition to the comparison between sampled ACC and fitted ACC, the exponential decay assumption can be verified with the factor $r$. First, $r$ from fitted autocorrelation coefficient is substituted into $R M S_{\text {predict }}(\mathrm{Eq} 4.4$, illustrated in Fig 4(b). Second, $r$ from fitted ACC predicts the sample variance underestimation ratio $\frac{N-r}{N-1}(\mathrm{Eq} 3.24$, given in Fig $4(\mathrm{c})$ It can be seen from Fig 4 that although 1 term exponential fitting does not predict ACC as well as 3 term fitting, it works well to predict the $R M S$ and predict the sample variance underestimation. Fig 4(b) also numerically verifies the unbiasedness of the sample mean since $R M S_{\text {predict }}$ stems from the variance of the estimator while $R M S$ stems from square error of the estimator. Their agreement demonstrates unbiasedness as given in Eq 3.9 .

\subsection{Independence of autocorrelation coefficients on generation size}

In Eq 4.4. if $q$ and $\rho_{0}$ as a function of generation size $s$ (number of histories per generation) are known, optimal values of $N$ and $s$ can be found to reach the minimum $R M S_{\text {predict }}^{2}$ given a total amount of work $N \cdot s$ or minimize $N \cdot s$ for a desired $R M S_{\text {predict }}^{2}$, assuming a stationary starting fission source. The optimization problem simplifies immensely if we assume that $q(s)$ and $\rho_{0}(s)$ are constant as a function of generation size, as evidenced by Fig 2(a). The ACCs estimated with Eq 3.25 for 3 different generation sizes almost overlap with one another. Two additional observations can be made supporting the assumption of constant $q(s)$ and $\rho_{0}(s)$ as a function of generation size. The observations will be presented in terms of $R M S_{\text {predict }}$ calculated from fitted ACCs and $R M S$ from the simulation. Define the total amount of work $N_{t}=N \cdot s$.

The first observation is that the $R M S$ vs $N_{t}$ curves of different generation sizes merge when $N_{t}$ approaches infinity (Fig 5). Without loss of generality, we investigate $R M S_{\text {predict }}^{2}$ of two simulations with generations size $s_{1}$ and $s_{2}$ respectively. The dependence of $R M S_{\text {predict }}^{2}$ on $s$ can be written explicitly

$$
\begin{aligned}
R M S_{\text {predict }}^{2}\left(N, \sigma^{2}, \rho_{0}, q\right) & =R M S_{\text {predict }}^{2}\left(N_{t} / s, \sigma(s)^{2}, \rho_{0}(s), q(s)\right) \\
& =\frac{\sigma^{2}(s)}{N_{t} / s}\left[1+2 \rho_{0}(s)\left(\frac{q(s)}{1-q(s)}-\frac{q(s)^{2}-q(s)^{N_{t} / s}}{(1-q(s))^{2} N_{t} / s}-\frac{q(s)+q(s)^{N_{t} / s}}{(1-q(s)) N_{t} / s}\right)\right]
\end{aligned}
$$

Regardless of the generation size, $s$, the $R M S_{\text {predict }}^{2}$ is unchanged as $N_{t}$ approaches infinity, as illustrated in Fig 5 and shown mathematically below

$$
\lim _{N_{t} \rightarrow \infty} \frac{R M S_{\text {predict }}^{2}\left(N_{t} / s_{1}, \sigma^{2}\left(s_{1}\right), \rho_{0}\left(s_{1}\right), q\left(s_{1}\right)\right)}{R M S_{\text {predict }}^{2}\left(N_{t} / s_{2}, \sigma^{2}\left(s_{2}\right), \rho_{0}\left(s_{2}\right), q\left(s_{2}\right)\right)}=1, \forall s_{1}, s_{2}
$$

Eq4.7 simplifies to Eq 4.8 after substituting Eq 4.6 and taking the limit

$$
\frac{s_{1}\left(1-q\left(s_{2}\right) \sigma^{2}\left(s_{1}\right)\left(1-q\left(s_{1}\right)+2 q\left(s_{1}\right) \rho_{0}\left(s_{1}\right)\right.\right.}{s_{2}\left(1-q\left(s_{1}\right) \sigma^{2}\left(s_{2}\right)\left(1-q\left(s_{2}\right)+2 q\left(s_{2}\right) \rho_{0}\left(s_{2}\right)\right.\right.}=1, \forall s_{1}, s_{2}
$$

where $\sigma^{2}\left(s_{1}\right)$ is the variance of the sample obtained from $N_{t} / s_{1}$ generations. At each generation, $X_{i}$ is calculated from the tally average over the $s_{1}$ neutrons. Thus, if the variance of the tally for one neutron is $c$ and the neutrons are independent, $\sigma^{2}\left(s_{1}\right)$ becomes $c / s_{1}$. In reality, the $s_{1}$ neutrons are not necessarily 

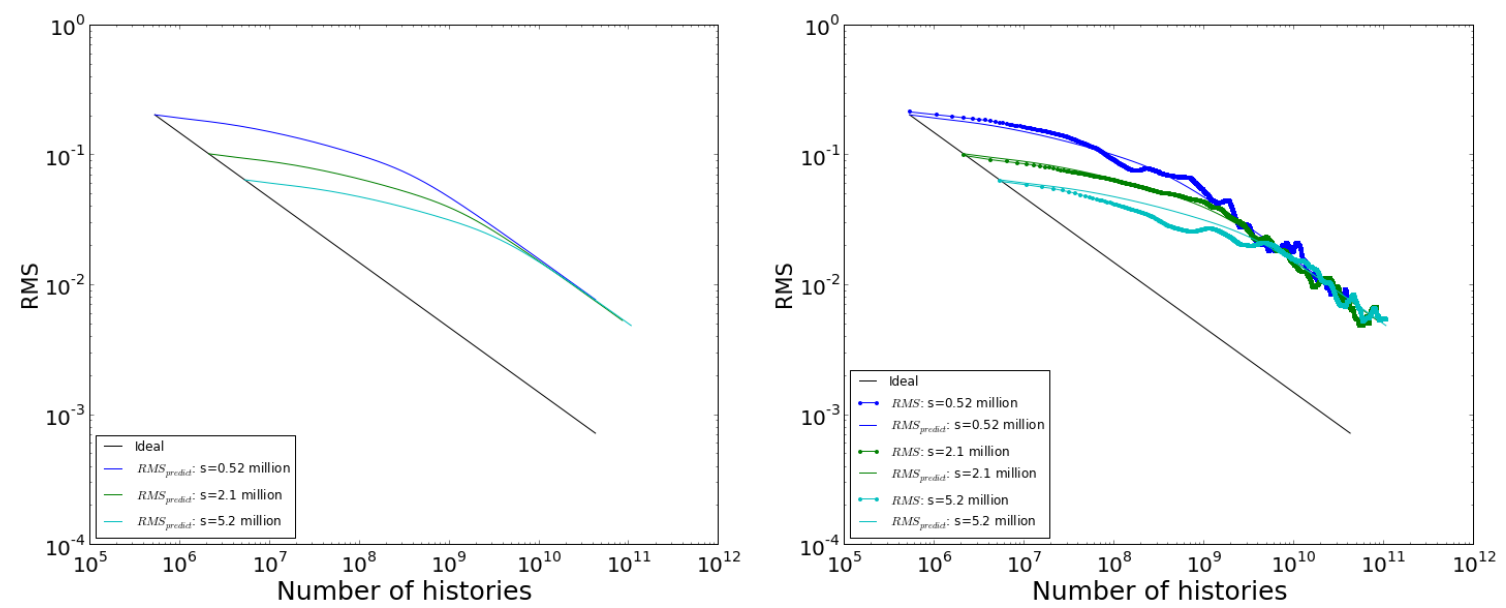

Figure 5: RMS $-N_{t}$ curves merge; left: prediction with fitted ACC; right: with RMS data from MC simulation

independent because neutrons generated from the same fission site are correlated. However, it is still reasonable to assume $\sigma^{2}\left(s_{1}\right) \propto \frac{1}{s_{1}}$

$$
\sigma\left(s_{1}\right)^{2}=\frac{c}{s_{1}}, \sigma\left(s_{1}\right)^{2}=\frac{c}{s_{2}}, \forall s_{1}, s_{2} \Rightarrow \sigma^{2}\left(s_{1}\right) s_{1}=\sigma^{2}\left(s_{2}\right) s_{2}=c
$$

From this, Eq4.8 simplifies to equation Eq4.10

$$
\frac{\rho_{0}\left(s_{1}\right) q\left(s_{1}\right)}{1-q\left(s_{1}\right)}=\frac{\rho_{0}\left(s_{2}\right) q\left(s_{2}\right)}{1-q\left(s_{2}\right)}, \forall s_{1}, s_{2}
$$

If $\rho_{0}(s)$ and $q(s)$ are constant as a a function of $s, \mathrm{Eq} 4.7$ and $\mathrm{Eq} 4.10$ hold, and the $R M S_{\text {predict }}^{2}$ curves will merge at large $N_{t}$ as observed in Fig 5

The second observation is that $R M S \times \sqrt{s}$ as a function of the number of generations, $N$, for different generation size overlap with each other (Fig 6). Similar to the above analysis, this dependence can be written explicitly

$$
\begin{aligned}
R M S_{\text {predict }}^{2}\left(N, \sigma^{2}, \rho_{0}, q\right) & =R M S_{\text {predict }}^{2}\left(N, \sigma(s)^{2}, \rho_{0}(s), q(s)\right) \\
& =\frac{\sigma^{2}(s)}{N}\left[1+2 \rho_{0}(s)\left(\frac{q(s)}{1-q(s)}-\frac{q(s)^{2}-q(s)^{N}}{(1-q(s))^{2} N}-\frac{q(s)+q(s)^{N}}{(1-q(s)) N}\right)\right]
\end{aligned}
$$

If $\rho_{0}(s)$ and $q(s)$ are constant as a function of generation size, $R M S_{\text {predict }}^{2}$ depends on $s$ only through the $\sigma^{2}$ term. Substituting Eq 4.9 into Eq 4.11 .

$$
\frac{R M S_{\text {predict }}^{2}\left(N, \sigma^{s}\left(s_{1}\right), \rho_{0}, q\right) s_{1}}{R M S_{\text {predict }}^{2}\left(N, \sigma^{s}\left(s_{2}\right), \rho_{0}, q\right) s_{2}}=1
$$

Therefore, the $R M S \times \sqrt{s}$ vs $N$ curves will overlap regardless of generation size as observed in Fig 6 .

In this section, it was numerically verified that $\rho_{0}$ and $q$ are constant as a function of generation size by directly investigating ACCs and observing the convergence behavior of $R M S$. This can also be supported by Yamamoto, et al. [6] and Sutton [8]'s work on predicting underestimation of variance, where the underestimate ratio is insensitive to generation size. The underestimation ratio in this paper is represented as $1+2 \rho_{0} \frac{q}{1-q}$ according to $\mathrm{Eq} 4.4$ and its insensitivity to generation size is consistent with the insensitivity of ACCs.

\section{Optimization}

When performing large Monte Carlo simulations, restrictions often come in the form of total runtime which is proportional to the total number of histories $N_{t}$. Alternatively, reactor simulations often seek answers to a known accuracy [18], thus leading to two possible type of optimizations: 

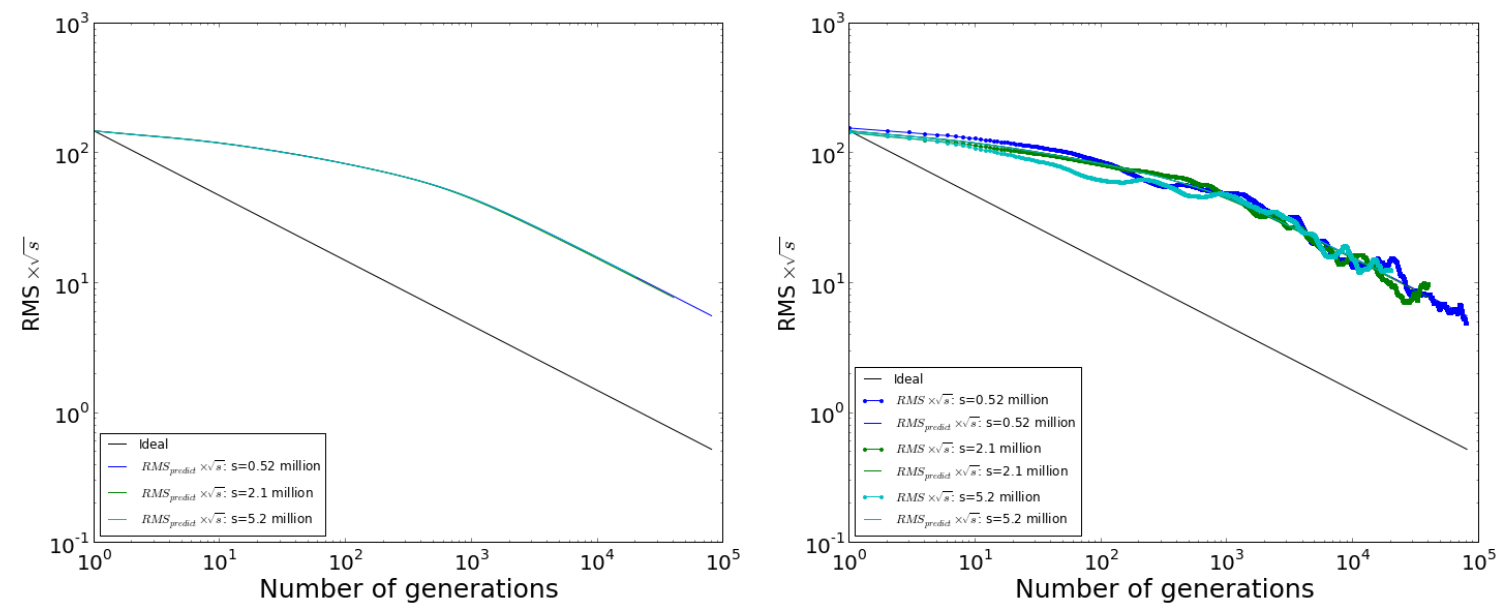

Figure 6: $R M S \times \sqrt{s}-N$ curves overlap; left: prediction with fitted ACC; right: with RMS data from $M C$ simulation

1. given a total computation amount $N_{t}$, find $N$ and $s$ to minimize $R M S_{\text {predict }}$

2. given desired $R M S_{\text {predict }}$, find $N$ and $s$ to minimize $N_{t}$

In this analysis, we assume that we are starting from a stationary source. Obviously, obtaining a stationary source for large correlated problems can be quite expensive, but it is assumed that this portion of the work can be greatly accelerated by other schemes currently in development [4] 10, 11, 12, 13, 14, [15.

Starting from Eq4.4 and re-writing it in terms of $N$ and $s$

$$
R M S_{\text {predict }}^{2}(N, s)=\frac{c}{N s}\left[1+2 \rho_{0}\left(\frac{q}{1-q}-\frac{q^{2}-q^{N}}{(1-q)^{2} N}-\frac{q+q^{N}}{(1-q) N}\right)\right] \equiv \frac{c}{N s} f(N)
$$

The optimization can be performed in the $N$ vs $s$ plane but can be $\operatorname{simplified~in~the~} \log N$ vs $\log s$ plane since the fixed $N \times s$ constraint becomes a straight line with slope -1 . Using parameters $c, q$ and $\rho_{0}$ evaluated from the cube problem with a $16 \times 16 \times 16$ mesh, $R M S_{\text {predict }}$ is plotted in Fig 7 To perform optimization of type 1 , we need to find the point at the $\log N+\log s=\log N_{t}$ curve where $R M S_{\text {predict }}$ is minimal. To perform optimization of type 2 , we need to find the point at the $R M S_{\text {predict }}(N, s)=R M S_{\text {goal }}$ curve where $N \times s$ is minimal.

Two equal- $R M S_{\text {predict }}$ curves are plotted in Fig $7(\mathrm{a})$ using thick black lines. One important feature of these curves in $\log N$ vs $\log s$ plane that affects optimization can be stated as such

$$
\frac{d \log s}{d \log N} \in(-1,0)
$$

The slope of the equal- $R M S_{\text {predict }}$ curves can be evaluated as

$$
\frac{d \log s}{d \log N}=-\frac{\frac{\partial R M S_{\text {predict }}^{2}(N, s)}{\partial \log N}}{\frac{\partial R M S_{\text {predict }}^{2}(N, s)}{\partial \log s}}=-\frac{N}{s} \frac{\frac{\partial R M S_{p r e d i c t}^{2}(N, s)}{\partial N}}{\frac{\partial R M S_{p r e d i c t}^{2}(N, s)}{\partial s}}=-\frac{N}{s} \frac{\frac{\partial R M S_{p r e d i c t}^{2}(N, s)}{\partial N}}{-\frac{c}{N s^{2}} f(N)}=N \frac{\frac{\partial R M S_{\text {predict }}^{2}(N, s)}{\partial N}}{R M S_{\text {predict }}^{2}(N, s)}
$$

Since $R M S_{\text {predict }}^{2}(N, s)>0, \mathrm{Eq} 5.2$ is equivalent to

$$
N \frac{\partial R M S_{\text {predict }}^{2}(N, s)}{\partial N}+R M S_{\text {predict }}^{2}(N, s)>0
$$

which can be related with $\frac{d R M S_{\text {predict }}^{2}\left(N_{t}, s\right)}{d s}$ for a fixed $N_{t}$ through 


$$
\begin{aligned}
\frac{d R M S_{\text {predict }}^{2}\left(N_{t}, s\right)}{d s} & =\frac{c}{N_{t}} \frac{d f(N)}{d s}=\frac{c}{N_{t}} \frac{d f(N)}{d N} \frac{d N}{d s}=-\frac{c}{N_{t}} \frac{d f(N)}{d N} \frac{N_{t}}{s^{2}} \\
& =-\left(\frac{\partial R M S_{\text {predict }}^{2}(N, s)}{\partial N}-\frac{\partial \frac{c}{N_{t}}}{\partial N} f(N)\right) \frac{N_{t}}{s^{2}} \\
& =-\left(\frac{\partial R M S_{\text {predict }}^{2}(N, s)}{\partial N}+\frac{c}{N^{2} s} f(N)\right) \frac{N}{s} \\
& =-\left(N \frac{\partial R M S_{\text {predict }}^{2}(N, s)}{\partial N}+R M S_{\text {predict }}^{2}(N, s)\right) \frac{1}{s}
\end{aligned}
$$

The monotonicity of $R M S_{\text {predict }}^{2}(s)$ for a fixed $N_{t}$ can be easily proven by writing $R M S_{\text {predict }}^{2}(\mathrm{Eq} 4.4)$ as a function of $N_{t}$ and $s$ and taking the derivative with respect to $s$ as done in Eq 5.6 and Eq 5.7

$$
R M S_{\text {predict }}^{2}\left(N_{t}, s\right)=\frac{c}{N_{t}}\left[1+2 \rho_{0}\left(\frac{q}{1-q}-\frac{q^{2}-q^{N_{t} / s}}{(1-q)^{2} N_{t} / s}-\frac{q+q^{N_{t} / s}}{(1-q) N_{t} / s}\right)\right]
$$

where $c$ is the constant first introduced in $\mathrm{Eq} 4.9$

$$
\frac{d R M S_{\text {predict }}^{2}(s)}{d s}=-\frac{2 q \rho_{0}}{N_{t}(1-q)^{2} s}\left(s-q^{N_{t} / s} s+N_{t} q^{N_{t} / s} \log (q)\right)<0
$$

where the sign of the derivative is always negative.

Therefore $R M S_{\text {predict }}$ decreases with generation size $s$. This trend can also be easily observed from Fig 5 . For the same number of total histories, before reaching the asymptotic regime, a larger $s$ always produces a lower $R M S$, thus indicating a preference for large generation size.

Validity of Eq 5.2 has been demonstrated with Eq 5.7 and Eq 5.5. Defining the tangent vector, $\tau$, and the gradients of $N_{t}$ and $R M S_{\text {predict }}^{2}$ can facilitate the explanation of both optimization types.

$$
\begin{aligned}
\nabla R M S_{\text {predict }}^{2} & =\left(\frac{\partial R M S_{\text {predict }}^{2}(N, s)}{\partial \log N}, \frac{\partial R M S_{\text {predict }}^{2}(N, s)}{\partial \log s}\right) \equiv\left(g_{1}, g_{2}\right) \\
\boldsymbol{\tau}\left(R M S_{\text {predict }}^{2}\right) & =\left(1,-\frac{g_{1}}{g_{2}}\right) \\
\boldsymbol{\nabla} N_{t} & =(1,1) \\
\boldsymbol{\tau}\left(N_{t}\right) & =(1,-1)
\end{aligned}
$$

\subsection{Type 1}

Optimization of type 1 is to find the lowest $R M S_{\text {predict }}$ on the straight line of constant $N_{t}\left(N \times s=10^{5}\right.$ in Fig $7(\mathrm{a})$. From Fig $7(\mathrm{a})$, this clearly indicates that the lowest $R M S_{\text {predict }}$ predict is obtained with the fewest number of generations. This can also be shown mathematically by taking the tangent of the $N_{t}$ line multiplied by the gradient of $R M S_{\text {predict }}^{2}$

$$
\boldsymbol{\tau}\left(N_{t}\right) \cdot \nabla R M S_{\text {predict }}^{2}>0
$$

which is guaranteed since $g_{1}<0, g_{2}<0$ and $-1<-\frac{g_{1}}{g_{2}}<0$.

\subsection{Type 2}

Optimization of type 2 is to find the lowest $N_{t}$ for a desired $R M S_{\text {predict }}\left(R M S_{\text {predict }}^{2}=1.88 \times 10^{-6}\right.$ in Fig 7(b) . Moving from right to left in Fig $7(\mathrm{~b})$ the desired $R M S_{\text {predict }}$ intersects the $N_{t}=10^{6}$ line followed by the better case on the left with $N_{t}=19952$. This can also be shown mathematically by looking at the tangent of the equal- $R M S_{\text {predict }}$ curve.

$$
\boldsymbol{\tau}\left(R M S_{\text {predict }}^{2}\right) \cdot \nabla N_{t}>0
$$

This expression indicates that when moving tangentially from the $R M S_{\text {predict }}^{2}$ curve, $N_{t}$ always increases.

Everything we have done so far indicates a need for large $s$ and no generations which is not always practical. Large generation size will impact the inactive generations and can require an excessive amount of memory for the storage of the fission source. Additionally, common practice requires the definition of confidence intervals often done over generations, which will be discussed in the next section. 


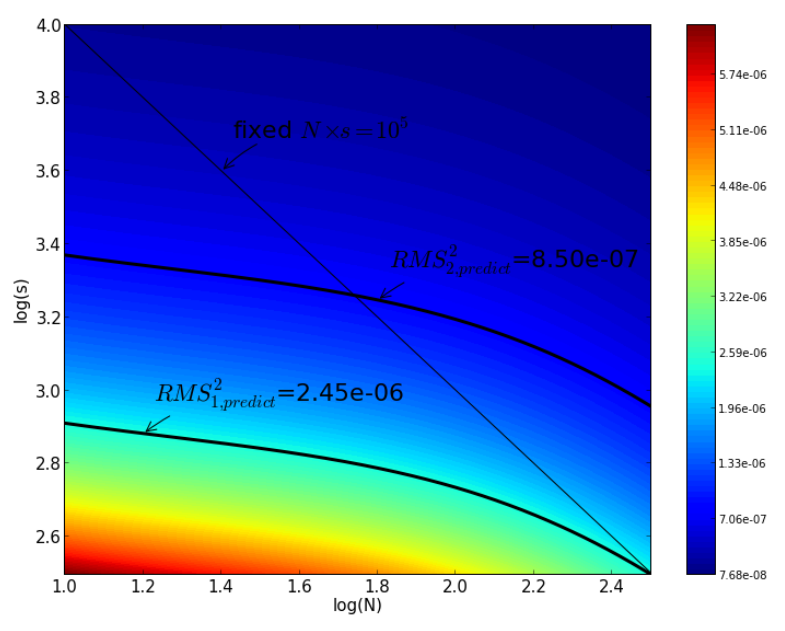

(a) Optimization given $N_{t}$

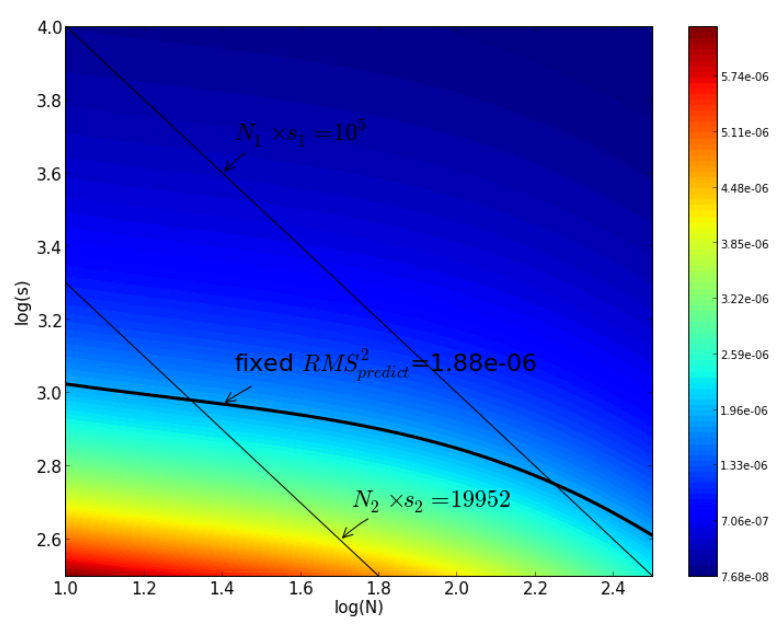

(b) Optimization given $R M S_{\text {predict }}$

Figure 7: Two types of optimization

\section{Interval Estimation}

This section gives a brief review of interval estimate of uncorrelated samples and then discusses the extension to correlated samples. The basic idea of interval estimate is to construct a pivot quantity $Q$, which depends on the statistic $T$ calculated from a sample and the parameter $\theta$ to be estimated but whose distribution does not depend on $\theta$ as expressed in the following equation

$$
\operatorname{Pr}\left(t_{1}<Q(T, \theta)<t_{2}\right)=P
$$

which can be converted to

$$
\begin{array}{r}
\operatorname{Pr}\left(f_{1}\left(T, t_{1}\right)<\theta<f_{2}\left(T, t_{2}\right)=P\right. \\
\text { or } \operatorname{Pr}\left(f_{1}\left(T, t_{2}\right)<\theta<f_{2}\left(T, t_{1}\right)=P\right.
\end{array}
$$

since the distribution of $Q$ does not depend on $\theta$, neither do $t_{1}$ and $t_{2}$. Therefore $\left(f_{1}\left(T, t_{1}\right), f_{2}\left(T, t_{2}\right)\right)$ or $\left(f_{1}\left(T, t_{2}\right), f_{2}\left(T, t_{1}\right)\right)$ is the confidence interval of $\theta$ with probability $P$.

Suppose in generation $i$, the $s$ histories provide tallies of quantity $\mathrm{x},\left\{x_{i, 1}, \cdots, x_{i, s}\right\}$ from which a generation mean is defined

$$
X_{i}=\frac{1}{s} \sum_{j=1}^{s} x_{i, j}
$$

Additionally, for $N$ generations with sample $\left\{X_{1}, X_{2}, \cdots, X_{N}\right\}$, the overall simulation mean is given by

$$
\bar{X}=\frac{1}{N} \sum_{i=1}^{N} X_{i}
$$

which is an estimate of the expectation of the true mean $\mu$. If the neutrons within a generation are assumed independent of each other, thus tallied quantities $\left\{x_{1}, \cdots, x_{s}\right\}$ are independent. According to the central limit theorem, the average over these neutrons $\left(X_{i}\right)$ converges to a normal distribution,

$$
X_{i} \stackrel{d}{\Rightarrow} N\left(\mu, \sigma^{2}\right)=N\left(\mu, \frac{c}{s}\right)
$$

where $\stackrel{d}{\Rightarrow}$ denotes convergence in distribution, $\sigma^{2}$ is the variance of $X$, and $c$ is the variance of $x$.

\subsection{Uncorrelated Generations}

This subsection reviews the confidence intervals that would be obtained if generation correlation is neglected. If the sample $\left\{X_{1}, \cdots, X_{N}\right\}$ is uncorrelated, an interval estimate can be obtained by 
constructing a Student's t distribution pivot. For i.i.d random variables $X_{i} \sim N\left(\mu, \sigma^{2}\right)$, if $\mu$ is estimated with $\mathrm{Eq} 6.4$ and $\sigma^{2}$ with $\widehat{\sigma^{2}}(\mathrm{Eq} 3.17$, the pivot can be written as

$$
Q_{U G 1}=\frac{\bar{X}-\mu}{\sqrt{\widehat{\sigma^{2}} / N}} \sim t_{N-1}
$$

where $t_{N-1}$ does not dependent on $\mu$ and $\sigma^{2}[19$.

Substituting $Q_{U G 1}, \theta=\mu, t_{1}=-t_{N-1, \alpha / 2}, t_{2}=t_{N-1, \alpha / 2}$ and $P=1-\alpha$ in Eq 6.1 provides the uncorrelated generation statistics confidence interval (UGCI) of $\mu$ as

$$
U G C I_{g}=\bar{X} \pm t_{N-1, \alpha / 2} \frac{\sqrt{\widehat{\sigma^{2}}}}{\sqrt{N}}
$$

where $t_{N-1, \alpha / 2}$ is the $\alpha / 2$ quantile of student's t distribution of degrees of freedom $N-1$ and $\widehat{\sigma^{2}}$ is the estimator of $\sigma^{2}$ (Eq 3.17). $U G C I_{g}$ only requires a large generation size to approximate $X_{i}$ by a normal distribution but applies to any generation number $N>2$. When the generation number, $N$, approaches infinity, the limit of $U G C I$ is

$$
U G C I_{g}=\bar{X} \pm Z_{\alpha / 2} \frac{\sqrt{\widehat{\sigma^{2}}}}{\sqrt{N}}
$$

This can be obtained by taking the limit of $t_{N-1, \alpha / 2}$ to $Z_{\alpha / 2}$.

Alternatively, $\sigma^{2}$ can be calculated from $\hat{c}$ (history based variance estimate of $c$ ), which does not require generation statistics over the sample $\left\{X_{1}, \cdots, X_{N}\right\}$. Therefore $\sigma^{2}$ can be assumed to be known and a normal pivot can be constructed

$$
Q_{U G 2}=\frac{\bar{X}-\mu}{\sqrt{\sigma^{2}} / \sqrt{N}} \stackrel{d}{\Rightarrow} N(0,1)
$$

where

$$
\begin{aligned}
\sigma^{2} & =\frac{\hat{c}}{s} \\
\hat{c} & =\frac{1}{N} \sum_{i=1}^{N} \frac{1}{s-1} \sum_{j=1}^{s}\left(x_{i, j}-X_{i}\right)^{2} \equiv \widehat{\sigma^{2}} h
\end{aligned}
$$

Each generation gives an estimate of $c$, while $\hat{c}$ is the average.

Substituting $Q_{U G 2}, \theta=\mu, t_{1}=-Z_{\alpha / 2}, t_{2}=Z_{\alpha / 2}$ and $P=1-\alpha$ in Eq 6.1 gives the uncorrelated generation confidence interval of $\mu$ as

$$
U G C I_{h}=\bar{X} \pm Z_{\alpha / 2} \frac{\sqrt{\widehat{c}}}{\sqrt{N s}}
$$

\subsection{Correlated Generations}

Since the generation results are correlated with each other, it is impossible to construct the traditional student's t-distribution pivot to perform interval estimate. If the sample $\left\{X_{1}, \cdots, X_{N}\right\}$ is viewed as a Markov Chain, as $N$ approaches infinity, the central limit theorem of Markov Chain holds [20] 21] and a normal distribution pivot can be constructed for interval estimates. More generally, Ibragimov 22] proved a variant of the central limit theorem for stationary sequence with weak dependence $\left(\rho_{k} \rightarrow 0\right.$ as $k \rightarrow \infty)$ :

$$
Q_{C G}=\frac{\bar{X}-\mu}{\sigma\left(\sum_{i=1}^{N} X_{i}\right) / N} \stackrel{d}{\Rightarrow} N(0,1)
$$

Following the procedure in section 3.1 it can be shown

$$
\sigma\left(\sum_{i=1}^{N} X_{i}\right)=\sqrt{\sigma^{2} N r}
$$




\begin{tabular}{|c|c|c|}
\hline & Uncorrelated generations & Correlated generations \\
\hline generation statistics (unknown variance) & $\frac{2 t_{N-1, \alpha / 2}}{\sqrt{N}} \sqrt{\widehat{\sigma^{2}}}\left(U G C I_{g}, \mathrm{Eq} 6.8\right)$ & $\frac{2 Z_{\alpha / 2}}{\sqrt{N}} \sqrt{\widehat{\sigma^{2}}} \sqrt{r}\left(C G C I_{g}, \mathrm{Eq} 6.16\right)$ \\
\hline history statistics (known variance) & $\frac{2 Z_{\alpha / 2}}{\sqrt{N}} \sqrt{\frac{\hat{c}}{s}}\left(U G C I_{h}, \mathrm{Eq} 6.11\right)$ & $\frac{2 Z_{\alpha / 2}}{\sqrt{N}} \sqrt{\frac{\hat{c}}{s}} \sqrt{r}\left(C G C I_{h}, \mathrm{Eq} 6.19\right.$ \\
\hline
\end{tabular}

where $r$ is defined in $\mathrm{Eq} 3.7$. Similar to the analysis of uncorrelated generations, there are two methods to obtain confidence interval from $\mathrm{Eq} 6.12$ the first estimates $\sigma\left(\sum_{i=1}^{N} X_{i}\right)$ from generation statistics and the second assumes $\sigma\left(\sum_{i=1}^{N} X_{i}\right)$ is known from history statistics.

If $\sigma^{2}$ is estimated from generation statistics substituted into the original pivot in Eq6.12. Slutsky's theorem [23] determines the distribution of pivot $Q_{C G 1}$ :

$$
\begin{aligned}
\sqrt{\widehat{\sigma^{2}} N r} \stackrel{P}{\Rightarrow} \sigma\left(\sum_{i=1}^{N} X_{i}\right) \\
Q_{C G 1}=\frac{\bar{X}-\mu}{\sqrt{\widehat{\sigma^{2}} r} / \sqrt{N}} \stackrel{d}{\Rightarrow} N(0,1)
\end{aligned}
$$

Substituting $Q_{C G 1}, \theta=\mu, t_{1}=-Z_{\alpha / 2}, t_{2}=Z_{\alpha / 2}$ and $P=1-\alpha$ in Eq 6.1 provides the correlated generation confidence interval $(C G C I)$ of $\mu$ as

$$
C G C I_{g}=\bar{X} \pm Z_{\alpha / 2} \frac{\sqrt{\widehat{\sigma^{2}}}}{\sqrt{N}} \sqrt{r}
$$

where $\widehat{\sigma^{2}}$ is the estimator of $\sigma^{2}$ from generation-based statistics

$$
\widehat{\sigma^{2}}=\frac{1}{N-r} \sum_{i=1}^{N}\left(X_{i}-\bar{X}\right)^{2} \equiv \widehat{\sigma^{2}} g
$$

Alternatively, the denominator of $Q_{C G}$ in Eq 6.12 can be approximated from history statistics. Thus the pivot can be written as

$$
Q_{C G 2}=\frac{\bar{X}-\mu}{\sqrt{\sigma^{2} / N r}} \stackrel{d}{\Rightarrow} N(0,1)
$$

Substituting $Q_{C G 2}, \theta=\mu, t_{1}=-Z_{\alpha / 2}, t_{2}=Z_{\alpha / 2}$ and $P=1-\alpha$ in Eq 6.1 provides the correlated generation confidence interval of $\mu$ as

$$
C G C I_{h}=\bar{X} \pm Z_{\alpha / 2} \frac{\sqrt{\sigma^{2}}}{\sqrt{N}} \sqrt{r}
$$

where $\sigma^{2}$ can be approximated as given in $\mathrm{Eq} 6.10$. Since each generation gives an unbiased estimate of $c$, although generations are correlated, their average is unbiased.

Table 2 summarizes confidence interval options from history statistics and generation statistics using the same total number of simulated neutrons. Generation statistics are based on tallies from $N$ generations with size $s$ while history statistics are based on tallies from all generation with $N \times s$ neutrons. $\bar{X}$ will be the same no matter whether history statistics or generation statistics are used and the confidence intervals are symmetric about the common center, therefore, only the size of confidence intervals are compared.

Table 2 also shows that the confidence intervals are larger by a factor of $\sqrt{r}$ in the presence of generation-to-generation correlation. The bias of the variance estimator as noted in Eq 3.24 can be 
neglected since $r \ll N$. Since the confidence intervals are derived from pivots where $N \rightarrow \infty$, the asymptotic value of $r$ can be used to calculate confidence intervals. Using the simple benchmark with $16 \times 16 \times 16$ mesh, $\sqrt{r}$ approaches 8 asymptotically. The factor $r$ is defined in Eq 3.7 and is a function of the number of generations $N$. Additionally, it can be observed in Table 2 that history statistics and generation statistics will give the same confidence interval as long as both $\widehat{\sigma^{2}}$ and $\hat{c} / s$ estimate the variance of $X\left(\sigma^{2}\right)$ accurately.

\subsection{Numerical Example}

\subsubsection{Method 1: Asymptotic Confidence Interval with large $N$}

The simplest way to obtain confidence intervals is to run a large number of generations and assume $N$ to approach infinity such that $C G C I_{g}(\mathrm{Eq} 6.16)$ or $C G C I_{h}(\mathrm{Eq} 6.19)$ can be used directly. The problem is that there is no criterion to determine whether $N$ is large enough to validate the central limit theorem for correlated generation tallies or the asymptotic normal distribution of pivot $Q_{C G}(\mathrm{Eq} 6.12)$.

\subsubsection{Method 2: Asymptotic Confidence Interval without explicit $r$ dependence}

Using a batching algorithm enables evaluating the confidence interval without the need of $r$ [24] 25]. This method has been implemented in OpenMC [26], MCNP [27] and MC21[5]. The total number of active generations $N$ is divided into $N_{B}=N / B$ batches, where a batch consists of $B$ consecutive fission generations. The batch $k$ tally value is obtained as

$$
\mathcal{X}_{k}=\frac{1}{B} \sum_{i=(k-1) M+1}^{k M} X_{i}
$$

where $X_{i}$ is the generation $i$ tally value. Statistics over the sample $\left\{\mathcal{X}_{1}, \cdots, \mathcal{X}_{N_{B}}\right\}$ are performed to construct a pivot and obtain a confidence interval. According to Kelly et al, by making $B$ sufficiently large, the correlation between batches can be reduced to a negligible level. Therefore with a pivot similar to $Q_{U G 1}(\mathrm{Eq} 6.6)$, confidence interval in the form of $U G C I_{g}(\mathrm{Eq} 6.8)$ can be obtained by replacing $N$ with $N_{B}$, and $\widehat{\sigma^{2}}$ with $\widehat{\sigma_{\mathcal{X}}^{2}}$. This is denoted as $U B C I$ (Uncorrelated Batches Confidence Interval) and is evaluated as

$$
\begin{aligned}
U B C I & =\overline{\mathcal{X}} \pm \frac{t_{N_{B}-1, \alpha / 2}}{\sqrt{N_{B}}} \sqrt{\widehat{\sigma_{\mathcal{X}}^{2}}} \\
\widehat{\sigma_{\mathcal{X}}^{2}} & =\frac{1}{\left(N_{B}-1\right)} \sum_{k=1}^{N_{B}}\left(\mathcal{X}_{k}-\overline{\mathcal{X}}\right)^{2}
\end{aligned}
$$

$U B C I$ is consistent with $C G C I_{g}$ or $C G C I_{h}$. By definition of $\mathcal{X}(\mathrm{Eq} 6.20$

$$
\sigma_{\mathcal{X}}^{2}=\frac{\sigma^{2}}{B} r(B)
$$

where the dependence of $r$ on number of generations is recalled. Therefore $\widehat{\sigma_{\mathcal{X}}^{2}}$ along with $\sqrt{N_{B}}$ in $U G C I$ is consistent with $\sqrt{\frac{\widehat{\sigma^{2} r}}{N}}$ in $C G C I_{g}$ and $\sqrt{\frac{\hat{c} r}{s N}}$ in $C G C I_{h}$. They are all estimators of the variance of $\bar{X}$.

The main problem of this method is that there is no criterion to determine whether $B$ is sufficiently large to validate the independence between batches. Theory developed so far in this paper can be applied to provide a criterion with respect to the correlation between batches. Similarly to the analysis in Sec 3.1. covariance between batch $J$ and $K$ can be evaluated as

$$
\operatorname{Cov}\left[\mathcal{X}_{J}, \mathcal{X}_{K}\right]=\frac{\sigma^{2}}{B^{2}} \sum_{l=(\Delta-1) B+1}^{(\Delta+1) B-1}(\Delta B-|\Delta B-l|) \rho_{l}
$$

where $\Delta=|J-K| \geq 1$ is the batch lag, $\rho_{l}$ is the correlation coefficient between generations with lag $l$ as defined in Eq 3.6. The correlation coefficient between batches with lag $\Delta$ can be further obtained as

$$
\varrho_{\Delta}=\frac{\operatorname{Cov}\left[\mathcal{X}_{J}, \mathcal{X}_{K}\right]}{\sigma_{\mathcal{X}}^{2}}=\frac{1}{B r(B)} \sum_{l=(\Delta-1) B+1}^{(\Delta+1) B-1}(\Delta B-|\Delta B-l|) \rho_{l}
$$


With the verified assumption of exponential decay of $\rho_{l}$, batch correlation coefficient $\varrho_{\Delta}$ can be evaluated with the exponential decay parameters as introduced in Sec 4.1. With the one-term exponential form of $\rho_{k}, \varrho_{1}$ can be obtained as

$$
\varrho_{1}=\frac{q \rho_{0}\left(1-q^{B}\right)^{2}}{B(1-q)\left(2 \rho_{0} q-q+1\right)-2 q \rho_{0}\left(1-q^{B}\right)}
$$

Finally, given the exponential decay parameter of generation correlation coefficients $\rho_{k}$, the "sufficiently" large $B$ can be found through $\varrho_{1} \sim 0$.

\subsubsection{Method 3: Confidence Interval for finite $N$}

In addition to central limit theorem, distribution of pivots constructed at the first of this section can also be obtained with more assumptions. If the asymptotic distribution of $X(\mathrm{Eq} 6.5)$ is substituted into the pivot $Q_{C G 2}$, distribution of $Q_{C G 2}$ can be obtained for any $N$. It can be shown that for $N$ random variables $X_{i} \sim N\left(\mu, \sigma^{2}\right)$, their average is still normal.

Denote the $N$ Gaussian random variables as an $N$ dimension random vector $\vec{X} \sim(N(\vec{\mu}, \overline{\bar{\Sigma}})$, where $\overline{\bar{\Sigma}}$ is the covariance matrix with $\Sigma_{i, j}=\operatorname{Cov}\left[X_{i}, X_{j}\right]$. The characteristic function of the Gaussian random vector is

$$
\phi_{\vec{X}}(\vec{t})=E\left[e^{-i \vec{t}^{T} \vec{X}}\right]=e^{-i \vec{t}^{T} \vec{\mu}+\frac{1}{2} \vec{t}^{T} \overline{\bar{\Sigma}} \vec{t}}
$$

The average of the $N X$ 's can be denoted as

$$
\begin{aligned}
\bar{X} & =\vec{a}^{T} \vec{X} \\
\vec{a}^{T} & =\frac{1}{N}(1, \cdots, 1)
\end{aligned}
$$

Then the characteristic function of $\bar{X}$ can be found as

$$
\phi_{\bar{X}}(s)=E\left[e^{-i s \vec{a}^{T} \vec{X}}\right]=\phi_{\vec{X}}(s \vec{a})=e^{-i s \vec{a}^{T} \vec{\mu}+\frac{1}{2} s^{2} \vec{a}^{T} \overline{\bar{\Sigma}} \vec{a}}
$$

Therefore $\bar{X}$ is a Gaussian random variable with mean $\vec{a}^{T} \vec{\mu}=\mu$ and variance $\sigma^{2}(\bar{X})=\vec{a}^{T} \overline{\bar{\Sigma}} \vec{a}$. This leads to a Gaussian pivot for finite $N, Q_{N}$,

$$
Q_{N}=\frac{\bar{X}-\mu}{\sqrt{\sigma^{2} r(N)} / \sqrt{N}} \sim N(0,1)
$$

where $\sigma^{2}$ and $r(N)$ are assumed to be known and $\sigma^{2}$ can be evaluated with Eq 6.10. In comparison with $Q_{C G 2}$, distribution of $Q_{N}$ does not require large $N$ and the variance underestimate ratio need not take the asymptotic value. Substituting $Q_{N}, \theta=\mu, t_{1}=-Z_{\alpha / 2}, t_{2}=Z_{\alpha / 2}$ and $P=1-\alpha$ in Eq 6.1 provides the correlated generation confidence interval of $\mu$ as

$$
C G C I_{N}=\bar{X} \pm Z_{\alpha / 2} \frac{\sqrt{\hat{c}}}{\sqrt{N s}} \sqrt{r(N)}
$$

\subsubsection{Results}

This section compares variance estimators of $\bar{X}(\mathrm{Eq} 6.4$ ), as a proxy to confidence intervals. The variance estimators calculated from history statistics (Eq 6.10), generation statistics (Eq 6.17) and batch statistics $(\mathrm{Eq} 6.22$ are summarized below.

$$
\begin{aligned}
& \widehat{\sigma_{\bar{X}}^{2}}=\frac{\widehat{\sigma^{2}} h}{s N} r(N) \\
& {\widehat{\sigma_{\bar{X}}^{2}}}_{g}=\frac{\widehat{\sigma^{2}} g}{N} r(N) \\
& \widehat{\sigma_{\bar{X}}^{2} b}=\frac{\widehat{\sigma^{2}} \mathcal{X}}{N_{B}}
\end{aligned}
$$


The reference variance value can be calculated as

$$
\sigma_{\bar{X}}^{2}=\frac{\sigma^{2}}{N}=\frac{c}{N s}
$$

where $\sigma^{2}$ can be obtained analytically and $r$ can be approximated as described in sec 4.1. The assumption that the tallies obtained by neutrons in one generation are independent are not true in traditional source update methods due to the possibility of re-using source sites in a given generation leading to history-to-history correlations. This correlation invalidates the relation between history variance $c$ and generation variance $\sigma^{2}$ described by $\mathrm{Eq} 4.9$ but can be avoided by removing this source site multiplicity. In the simple cube problem, sites can be re-sampled from a uniform distribution (exact solution), but more generally sites could be replaced by source sites from a previous generation.

Under the assumption of independent history tallies, the analytical variance of the fission tally is

$$
c=\frac{P_{f}}{M^{2}\left(1-P_{s}\right)^{2}}\left(P_{c}+\left(1-P_{s}\right)(M-1)\right.
$$

where $M$ is the number of meshes in the cube, and the fission probability $P_{f}$, scattering probability $P_{s}$ and capture probability $P_{c}$ can be calculated from the cross section parameters in Table 1 . Knowing $c$ and approximating $r(N), \mathrm{Eq} 6.36$ provides a reference value of the true variance.

Sixty independent simulations were performed using 10,000 generations of 983,040 neutrons per generation. Analog fission rates were tallied on $4 \times 4 \times 4$ mesh and results compared on a single tally region. The factor $r(N)$ is calculated following the procedure described in Sec 4.1 and assumed to be known.

The variance using the three methods for the 60 simulations are presented in Fig 8 , The batch statistics results were produced assuming batch groupings of $B=200$. The first observation in Fig 8 is that using uncorrelated statistics leads to underestimating the variance by a factor of 2 . This figure also demonstrates consistency between the three proposed methods for correlated statistics. The variance estimates from history and generation statistics are closely distributed around the theoretical value, however, batch statistics presents much greater dispersion. Appendix A demonstrates that history statistics will provide an estimation with a smaller uncertainty than generation-based estimates and that generation-based estimates provide an estimatation with a smaller uncertainty than batch-based variance estimates. This can be observed in Fig 8 and Fig 9 . For this particular problem, $B=200$ might be an overkill. The large $B$ leads to smaller number of batches, $N_{B}$, and further increases the uncertainty of the estimator $\widehat{\sigma_{\mathcal{X}}^{2}}$. If $B$ is instead set to 10 , the dispersion of $\widehat{\sigma_{\mathcal{X}}^{2}}$ becomes, as expected, much narrower but the average value deviates from the reference value (Fig $10(\mathrm{a})$. The deviation can be viewed as an underestimation of the batch variance and attributed to correlation between batches. Reducing the batch size $B$ from 200 to 10 increased the batch correlation coefficient of lag 1 (Eq 6.26) from 0.0022 to 0.048. Therefore, calculating the batch correlation, the variance can be corrected by a factor $\frac{r(N)}{r(B)}$. This corrected estimator matches the reference value as shown in Fig 10(b)p.

From these results it can be seen that history statistics (once history-to-history correlation is eliminated) can provide a suitable and accurate estimate of the variance. Thus, in support of the optimization conclusion of Sec 5, few generations with large particle counts is a possibility to provide the lowest possible tally errors while still providing an accurate measure of the confidence intervals. One caveat to this approach is that it requires an approximation of the underestimation factor. The batching scheme avoids this need, but the run strategy favors multiple generations which was shown to be sub-optimal with respect to the convergence rate. Additionally, history statistics require performing accumulation of tallies after each history which can become computationally prohibitive when many tallies are needed.

\section{Conclusions}

This paper provides an analysis of the generation-to-generation correlations as observed when solving full core eigenvalue problems on PWR systems. Many studies have in the past looked at the impact of these correlations on reported variance and this paper extends the analysis to the observed convergence rate on the tallies, the effect of tally size and the effect of generation size. Since performing meaningful analysis on such a large problem is inherently difficult, a simple homogeneous reflective cube problem was developed that exhibits similar behavior to the full core PWR benchmark. The data in this problem was selected to match the dimensionality of the reactor problem and preserve the migration length 


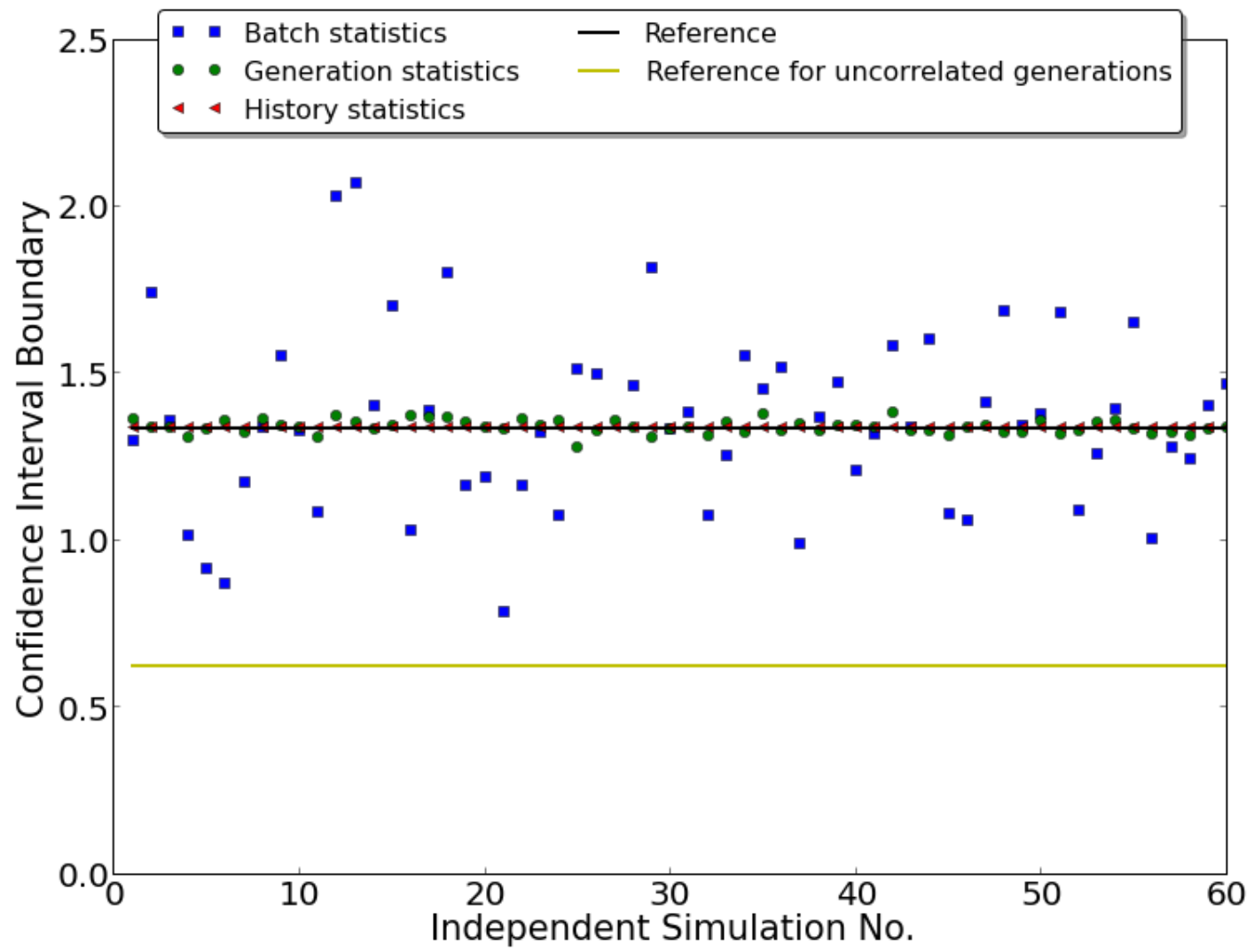

Figure 8: Comparison of CI evaluated from different methods

Batch statistics: Estimate $\widehat{\sigma_{\mathcal{X}}^{2}}$ in UBCI (Eq 6.21) with Eq 6.22.

Generation statistics: Estimate $\widehat{\sigma^{2}}$ in $C G C I_{h}(E q 6.16)$ with Eq 6.17 .

History statistics: Estimate $\widehat{c^{2}}$ in $C G C I_{N}$ (Eq 6.32) with $E q 6.10$ 


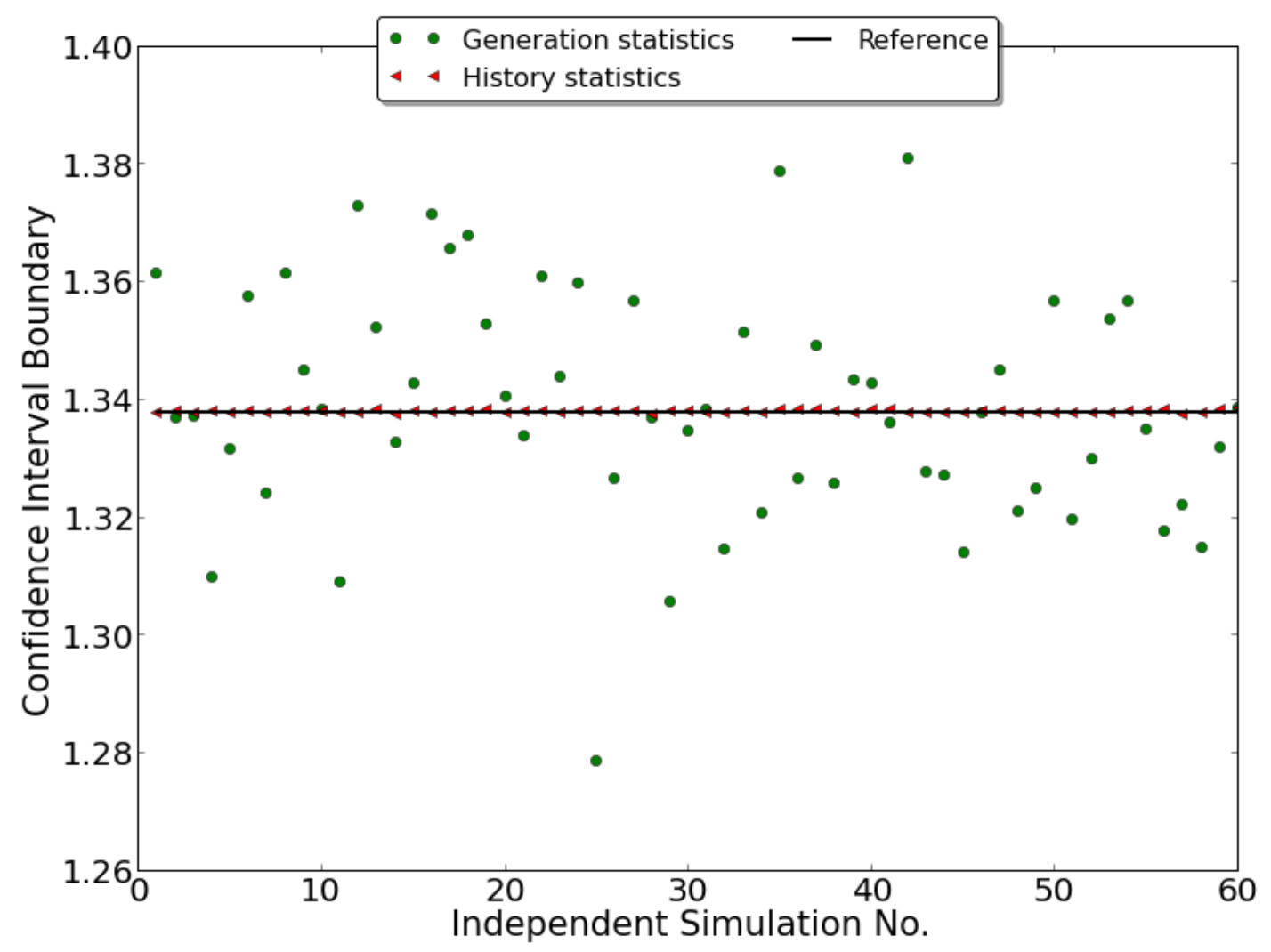

Figure 9: Comparison of CI: history and generation statistics

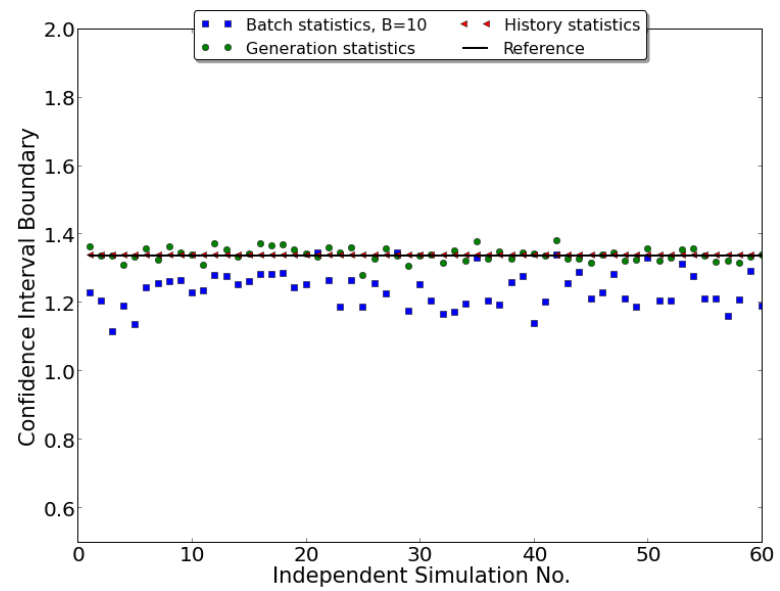

(a) $B=10, \widehat{\sigma_{\mathcal{X}}^{2}}$ uncorrected

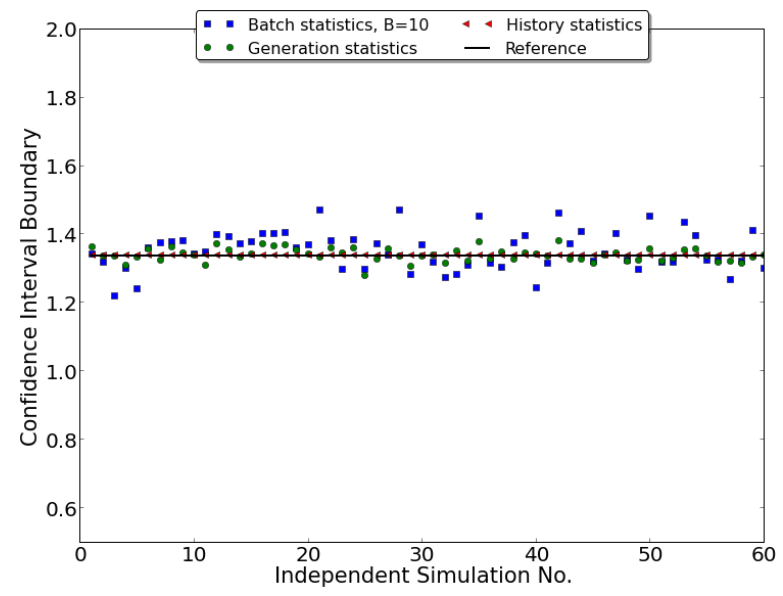

(b) $B=10, \widehat{\sigma_{\mathcal{X}}^{2}}$ corrected by $\frac{r(N)}{r(B)}$

Figure 10: Comparison of CI: batch statistics of different batch sizes 
traveled by neutrons. The reflective homogeneous nature of the problem also provides a simple analytical reference solution on which to determine convergence rates. The validity of this problem was verified by comparing the autocorrelation coefficients (ACCs) with those evaluated from the BEAVRS benchmark. One interesting observation can be made from this analysis, larger tally sizes exhibit greater generationto-generation correlation. This is supported by the convergence behavior of the power iteration method that is hindered more by the low frequency modes than the high ones.

Work by Sutton [8], Herman [4, and Yamamoto [6] has shown mathematically that the convergence rate of the tallies will deviate from $1 / \sqrt{N}$ in the presence of generation-to-generation correlation, but should eventually return to $1 / \sqrt{N}$. This paper demonstrates this fact once again in greater detail and provides supporting simulation results. Additionally, the ACCs were fitted using exponential terms in order to develop a predictive capability of the convergence rate. Thus, knowing the fit of the ACCs, one can determine the number of generations needed to achieve a desired level of accuracy. The fitting process and supporting simulations were also used to demonstrate the independence of ACCs on the generation size. Generation size will impact the level of accuracy but not the ACCs, thus only additional generations can overcome the correlation and allow convergence of the error to return to $1 / \sqrt{N}$. This paper demonstrates that realistic cases may require 1000's of generations before the convergence rate reaches the asymptotic behavior, thus indicating that commonly held belief of adding more generations provides little added value when true variance is measured.

This new information on ACCs and how they are impacted by generation size and number of generations brought a very interesting question on what is the optimal run strategy to minimize the tally errors. This analysis was made under the premise that a converged source distribution was provided using one of the many techniques currently under development [4 [10, $11,12,13,14,[15$. The analysis demonstrates that in the presence of correlations, it is beneficial to opt for large generation sizes and few generations. There are obvious limitations to such a running scheme like the impact on the inactive generations and storage requirements of fission sites. Additionally, common practice often requires many generations in order to provide suitable confidence intervals. This paper described three techniques to evaluate suitable confidence intervals in the presence of correlations, one based on using history statistics, one using generation statistics and one batching generations to reduce batch-to-batch correlation (5). The first two require some estimate of the variance underestimation factor evaluated from the ACCs, while batching can do without. The analysis indicates that history statistics are possible as long as one can remove history-to-history correlations often observed in current source update methods and would allow the use of a single generation if such a converged source bank could be generated. Batching will provide a good estimate of the true variance, but will suffer from poor convergence on highly-correlated problems since the scheme favors the use of many generations which is contrary to the optimal run strategy derived in this work.

Future work will focus on finding ways to estimate the ACCs (needed to calculate the underestimation factor) during the inactive generations such that few generations with large particle counts can be used to minimize the tally errors. Additionally, this work will be extended to reduce the history-tohistory correlation that was identified in Sec 6, which will in return reduce the overall correlation of the simulation.

\section{Acknowledgements}

This research was supported in part by the Consortium for Advanced Simulation of Light Water Reactors (CASL), an Energy Innovation Hub for Modeling and Simulation of Nuclear Reactors under U.S. Department of Energy Contract No. DE-AC05-00OR22725. The authors would also like to thank Eric Dumonteil, Andrea Zoia, Paul Romano and Tom Sutton for valuable discussions on this research. 


\section{Appendix A. Variance of Variance Estimators}

Sample $1\left\{x_{1}, x_{2}, \cdots, x_{M}\right\}$ is independently sampled from an identical distribution. Sample $2\left\{X_{1}, X_{2}, \cdots, X_{N}\right\}$ is obtained by splitting $\left\{x_{1}, \cdots, x_{M}\right\}$ into $N$ groups of size $s=M / N$ and calculating the average of each group.

$$
X_{i}=\frac{1}{s} \sum_{j=1}^{s} x_{(i-1) s+j}
$$

Variance of these two samples $\sigma_{1}^{2}$ and $\sigma_{2}^{2}$ satisfies

$$
\sigma_{2}^{2}=\frac{\sigma_{1}^{2}}{s}
$$

$\sigma_{1}^{2}$ can be estimated with

$$
S_{0}^{2}=\frac{1}{M-1} \sum_{i=1}^{M}\left(x_{i}-\bar{x}\right)^{2}
$$

Alternatively, $\sigma_{1}^{2}$ can be estimated by estimating the variance of each of the $N$ groups and averaging them

$$
S_{1}^{2}=\frac{1}{N} \sum_{i=1}^{N} \frac{1}{s-1} \sum_{j=1}^{s}\left(x_{(i-1) s+j}-X_{i}\right)^{2} \equiv \frac{1}{N} \sum_{i=1}^{N} S_{1, i}^{2}
$$

And $\sigma_{2}^{2}$ can be estimated with

$$
S_{2}^{2}=\frac{1}{N-1} \sum_{j=1}^{N}\left(X_{j}-\bar{X}\right)^{2}
$$

This section proves:

$$
\begin{aligned}
& \operatorname{Var}\left[S_{0}^{2}\right]<\operatorname{Var}\left[s S_{2}^{2}\right] \\
& \operatorname{Var}\left[S_{0}^{2}\right]<\operatorname{Var}\left[s \quad S_{2}^{2}\right]
\end{aligned}
$$

or equivalently

$$
\begin{aligned}
\operatorname{Var}\left[S_{0}^{2}\right] & <s^{2} \operatorname{Var}\left[S_{2}^{2}\right] \\
\operatorname{Var}\left[S_{1}^{2}\right] & <s^{2} \operatorname{Var}\left[S_{2}^{2}\right]
\end{aligned}
$$

Variance of sample variance can be evaluated as [23]

$$
\begin{aligned}
\operatorname{Var}\left[S_{0}^{2}\right] & =\frac{\mu_{4}(x)}{M}-\frac{\sigma(x)^{4}}{M} \frac{M-3}{M-1} \\
\operatorname{Var}\left[S_{1, i}^{2}\right] & =\frac{\mu_{4}(x)}{s}-\frac{\sigma(x)^{4}}{s} \frac{s-3}{s-1} \\
\operatorname{Var}\left[S_{2}^{2}\right] & =\frac{\mu_{4}(X)}{N}-\frac{\sigma(X)^{4}}{N} \frac{N-3}{N-1}
\end{aligned}
$$

where $\mu_{4}(x)$ is the $4^{\text {th }}$ central moment of $x, \sigma(x)^{4}$ is the square of variance of $x$. To compare Eq A.10 (or Eq A.11) with A.12 relation between $\mu_{4}(x)$ and $\mu_{4}(X), \sigma^{4}(x)$ and $\sigma^{4}(X)$ is needed. Since $X$ is the average of $s x$,

$$
\sigma^{4}(X)=\left(\sigma^{2}(X)\right)^{2}=\left(\frac{\sigma^{2}(x)}{s}\right)^{2}=\frac{\sigma^{4}(x)}{s^{2}}
$$

Relation between $\mu_{4}(X)$ and $\mu_{4}(x)$ can be derived as below, where $\mu=E[x]=E[X]=E[\bar{x}]=E[\bar{X}]$,

$$
\begin{aligned}
\mu_{4}(X) & =E\left[(X-\mu)^{4}\right]=E\left[\left(\frac{\sum_{j=1}^{s} x_{j}}{s}-\mu\right)^{4}\right] \\
& =\frac{1}{s^{4}} E\left[\left(\sum_{j=1}^{s}\left(x_{j}-\mu\right)\right)^{4}\right]
\end{aligned}
$$


For simplicity, define

$$
y_{i} \equiv x_{i}-\mu
$$

therefore

$$
E\left[y_{i}\right]=0
$$

$$
\begin{aligned}
s^{4} \mu_{4}(X) & =E\left[\left(\sum_{j=1}^{s} y_{j}\right)^{4}\right] \\
& =E\left[\sum_{j=1}^{s} y_{j}^{4}+3 \sum_{j \neq i} y_{j}^{2} y_{i}^{2}+4 \sum_{j \neq i} y_{j}^{3} y_{i}+6 \sum_{j \neq i \neq k} y_{j}^{2} y_{i} y_{k}+\sum_{j \neq i \neq k \neq l} y_{j} y_{i} y_{k} y_{l}\right] \\
& =\sum_{j=1}^{s} E\left[y_{j}^{4}\right]+3 \sum_{j \neq i} E\left[y_{j}^{2} y_{i}^{2}\right]+4 \sum_{j \neq i} E\left[y_{j}^{3} y_{i}\right]+6 \sum_{j \neq i \neq k} E\left[y_{j}^{2} y_{i} y_{k}\right]+\sum_{j \neq i \neq k \neq l} E\left[y_{j} y_{i} y_{k} y_{l}\right]
\end{aligned}
$$

Since the $x$ 's are independent,

$$
\begin{aligned}
s^{4} \mu_{4}(X) & =\sum_{j=1}^{s} E\left[y_{j}^{4}\right]+3 \sum_{j \neq i} E\left[y_{j}^{2}\right] E\left[y_{i}^{2}\right]+4 \sum_{j \neq i} E\left[y_{j}^{3}\right] E\left[y_{i}\right] \\
& +6 \sum_{j \neq i \neq k} E\left[y_{j}^{2}\right] E\left[y_{i}\right] E\left[y_{k}\right]+\sum_{j \neq i \neq k \neq l} E\left[y_{j}\right] E\left[y_{i}\right] E\left[y_{k}\right] E\left[y_{l}\right]
\end{aligned}
$$

The above equation can be simplified due to Eq A.16

$$
\begin{aligned}
s^{4} \mu_{4}(X) & =s E\left[y_{j}^{4}\right]+3 s(s-1) E\left[y_{j}^{2}\right] E\left[y_{i}^{2}\right] \\
& =s E\left[\left(x_{i}-\mu\right)^{4}\right]+3 s(s-1) E\left[\left(x_{j}-\mu\right)^{2}\right]^{2} \\
& =s \mu_{4}(x)+3 s(s-1) \sigma^{4}(x)
\end{aligned}
$$

Therefore $\mu_{4}(X)$ and $\mu_{4}(x)$ are related with

$$
\mu_{4}(X)=\frac{1}{s^{3}} \mu_{4}(x)+\frac{3(s-1)}{s^{3}} \sigma^{4}(x)
$$

With Eq A.13 and A.20, inequality to be proved (Eq A.8 can be equivalently transformed to

$$
\frac{\mu_{4}(x)}{N s}-\frac{\sigma^{4}(x)}{N s} \frac{N s-3}{N s-1}<\frac{\mu_{4}(x)}{N s}+\frac{3(s-1)}{N s} \sigma^{4}(x)-\frac{\sigma^{4}(x)}{N} \frac{N-3}{N-1}
$$

which is equivalent to

$$
2 \frac{\sigma^{4}(x)}{N s}\left(s-1+\frac{s}{N-1}-\frac{1}{N s-1}\right)>0
$$

It can be verified that if $s=1$, the two estimators $S_{0}^{2}$ and $s S_{2}^{2}$ have the same variance. As long as $s>1$, the above equation holds and $S_{0}^{2}$ has lower variance than $S_{2}^{2}$.

Similarly, with Eq A.13, Eq A.20 and $\operatorname{Var}\left[S_{1}^{2}\right]=\frac{\operatorname{Var}\left[S_{1, i}^{2}\right]}{N}$, inequality to be proved in Eq A.9 can be equivalently transformed to

$$
\frac{1}{N}\left(\frac{\mu_{4}(x)}{s}-\frac{\sigma^{4}(x)}{s} \frac{s-3}{s-1}\right)<\frac{\mu_{4}(x)}{N s}+\frac{3(s-1)}{N s} \sigma^{4}(x)-\frac{\sigma^{4}(x)}{N} \frac{N-3}{N-1}
$$

which is equivalent to

$$
2 \frac{\sigma^{4}(x)}{N s}\left(s-1+\frac{s}{N-1}-\frac{1}{s-1}\right)>0
$$


It can be verified that as long as $s>1$, the above equation holds and $S_{1}^{2}$ has lower variance than $S_{2}^{2}$.

When comparing variance estimated from history statistics and generation statistics, the uncertainty of the former one corresponds to $S_{1}^{2}$ the latter one corresponds to $S_{2}^{2}$. When comparing variance estimated from generation statistics and batch statistics, the uncertainty of the former one corresponds to $S_{0}^{2}$ the latter one corresponds to $S_{2}^{2}$. Therefore, history-based variance estimator always has lower uncertainty than generation-based variance estimator. And generation-based variance estimator always has lower uncertainty than batch-based variance estimator. 


\section{References}

[1] R. Brissenden, A. Garlick, Biases in the estimation of Keff and its error by Monte Carlo methods Annals of Nuclear Energy 13 (2) (1986) 63-83. doi:10.1016/0306-4549(86)90095-2

URL http://www.sciencedirect.com/science/article/pii/0306454986900952

[2] E. Dumonteil, F. Malvagi, A. Zoia, A. Mazzolo, D. Artusio, C. Dieudonné, C. De Mulatier, Particle clustering in Monte Carlo criticality simulations Annals of Nuclear Energy 63 (2014) 612-618. doi:10.1016/j.anucene.2013.09.008 URL http://linkinghub.elsevier.com/retrieve/pii/S0306454913004787

[3] B. R. Herman, B. Forget, K. Smith, P. K. Romano, T. M. Sutton, D. J. Kelly, B. N. Aviles, ANALYSIS OF TALLY CORRELATION IN LARGE LIGHT WATER REACTORS, in: PHYSOR 2014 - The Role of Reactor Physics toward a Sustainable Future, The Westin Miyako, Kyoto, Japan, 2014, pp. 1-14.

[4] B. R. Herman, Monte Carlo and Thermal Hydraulic Coupling using Low-Order Nonlinear Diffusion Acceleration , Ph.D. thesis, Massachusetts Institute of Technology (2014).

[5] S. C. W. Daniel J. Kelly, Thomas M. Sutton, MC21 ANALYSIS OF THE NUCLEAR ENERGY AGENCY MONTE CARLO PERFORMANCE BENCHMARK PROBLEM, in: PHYSOR 2012 Advances in Reactor Physics Linking Research, Industry, and Education, Knoxville, Tennessee, USA, 2002, pp. 1-17.

[6] A. Yamamoto, K. Sakata, T. Endo, Prediction on Underestimation of Variance for Fission Rate Distribution in Monte-Carlo Calculation, transaction of american nuclear society 110 (1) (2014) 515-518.

[7] A. Yamamoto, K. Sakata, T. Endo, Behavior of Higher Order Fission Source Distribution in Monte-Carlo Calculations, transaction of american nuclear society 109 (1) (2013) 1361-1364.

[8] T. M. Sutton, APPLICATION OF A DISCRETIZED PHASE SPACE APPROACH TO THE ANALYSIS OF MONTE CARLO UNCERTAINTIES, in: ANS MC2015 - Joint International Conference Mathematics and Computation (M\&C), Supercomputing in Nuclear Applications (SNA) and the Monte Carlo (MC) Method, no. Mc, Nashville, TN, 2015, pp. 1-17.

[9] K. Tuttelberg, J. Dufek, Neutron batch size optimisation methodology for Monte Carlo criticality calculations, Annals of Nuclear Energy 75 (2015) 620-626. doi:10.1016/j.anucene.2014.09.011. URL http://www.sciencedirect.com/science/article/pii/S0306454914004836

[10] M. J. Lee, H. G. Joo, D. Lee, K. Smith, Coarse mesh finite difference formulation for accelerated monte carlo eigenvalue calculation, Annals of Nuclear Energy 65 (2014) 101 -113. doi:http://dx.doi.org/10.1016/j.anucene.2013.10.025 URL http://www.sciencedirect.com/science/article/pii/S0306454913005550

[11] M. J. Lee, H. G. Joo, D. Lee, K. Smith, Monte Carlo reactor calculation with substantially reduced number of cycles, American Nuclear Society - ANS; La Grange Park, IL (United States), 2012.

[12] D. J. Kelly Iii, B. N. Aviles, B. R. Herman, MC21 analysis of the MIT PWR benchmark: Hot zero power results, American Nuclear Society - ANS; La Grange Park (United States), 2013.

[13] F. B. Brown, S. E. Carney, B. C. Kiedrowski, W. R. Martin, Fission matrix capability for MCNP, Part I - Theory, American Nuclear Society - ANS; La Grange Park (United States), 2013.

[14] S. E. Carney, F. B. Brown, B. C. Kiedrowski, W. R. Martin, Fission matrix capability for MCNP, Part II - Applications, American Nuclear Society - ANS; La Grange Park (United States), 2013.

[15] J. A. Willert, C. Kelley, D. Knoll, H. Park, A hybrid approach to the neutron transport k-eigenvalue problem using nda-based algorithms, in: Proceedings of International Conference on Mathematics and Computational Methods Applied to Nuclear Science and Engineering (M\&C 2013), Sun Valley, ID. American Nuclear Society, 2013.

[16] T. Ueki, F. B. Brown, D. K. Parsons, D. E. Kornreich, Autocorrelation and dominance ratio in monte carlo criticality calculations, Nuclear science and engineering 145 (3) (2003) 279-290.

[17] E. Dumonteil, F. Mavlagi, Variance estimation in monte carlo criticality simulations application to the axial study of a pwr cell with tripoli-4, in: Proceedings of the Second International Conference on Physics and Technology of Reactors and Applications (PHYTRA2), Fez, Morocco, 2011.

[18] Q. Shen, W. Boyd, B. Forget, K. Smith, Tally Precision Triggers for the OpenMC Monte Carlo Code, transaction of american nuclear society 112 (2015) 637-640.

[19] M. G. Kendall, S. Alan, THE ADVANCED THEORY OF STATISTICS, Vol. 2, CHARLES GRIFFIN. COMPANY LIMITED, 42. DRURY LANE. LONDON, W.C.2, 1961.

[20] G. L. Jones, On the Markov chain central limit theorem, Probability surveys 1 (299-320) (2004) 1-5. doi:10.1214/ 154957804100000051

[21] S. Brooks, A. Gelman, G. Jones, X.-L. Meng, Handbook of Markov Chain Monte Carlo, CRC press, 2011.

[22] I. A. Ibragimov, A note on the central limit theorems for dependent random variables, Theory of Probability \& Its Applications 20 (1) (1975) 135-141.

[23] G. Casella, R. L. Berger, Statistical inference, Vol. 2, Duxbury Pacific Grove, CA, 2002

[24] R. W. Conway, Some tactical problems in digital simulation, Management science 10 (1) (1963) $47-61$.

[25] E. Gelbard, R. Prael, Computation of standard deviations in eigenvalue calculations, Progress in Nuclear Energy 24 (1) (1990) 237-241.

[26] P. K. Romano, B. Forget, The OpenMC Monte Carlo particle transport code, Annals of Nuclear Energy 51 (2013) $274-281$.

[27] X.-. M. C. Team, Mcnp-a general monte carlo n-particle transport code, version 5, MCNP user manual. Report LA-UR-03-1987. Los Alamos, CA: Los Alamos National Laboratory 5. 